\title{
Ordered Phases of Itinerant Dzyaloshinsky-Moriya Magnets and Their Electronic Properties
}

\author{
Kwan-yuet Ho and T.R. Kirkpatrick \\ Institute for Physical Science and Technology, and Department of Physics, \\ University of Maryland, College Park, MD 20742, USA \\ Yan Sang and D. Belitz \\ Department of Physics, and Institute of Theoretical Science, \\ University of Oregon, Eugene, OR 97403, USA
}

(Dated: October 29, 2018)

\begin{abstract}
A field theory appropriate for magnets that display helical order due to the Dzyaloshinsky-Moriya mechanism, a class that includes MnSi and FeGe, is used to derive the phase diagram in a meanfield approximation. The helical phase, the conical phase in an external magnetic field, and recent proposals for the structure of the A-phase and the non-Fermi-liquid region in the paramagnetic phase are discussed. It is shown that the orientation of the helical pitch vector along an external magnetic field within the conical phase occurs via two distinct phase transitions. The Goldstone modes that result from the long-range order in the various phases are determined, and their consequences for electronic properties, in particular the specific heat, the single-particle relaxation time, and the electrical and thermal conductivities, are derived. Various aspects of the ferromagnetic limit, and qualitative differences between the transport properties of helimagnets and ferromagnets, are also discussed.
\end{abstract}

PACS numbers: 75.10.Hk; 75.20.En; 75.30.Ds

Contents

\section{Introduction}

A. Dzyaloshinsky-Moriya magnets

1. The phase diagram of $\mathrm{MnSi}$

2. Properties of the helical phase

B. Model, and energy scales

1. LGW functional

2. Length and energy scales

II. Phase Diagram

A. $O\left(g_{\mathrm{so}}^{0}\right)$ : Ferromagnet

B. $O\left(g_{\mathrm{so}}^{2}\right)$ : Helimagnet, conical phase

C. $O\left(g_{\mathrm{so}}^{2}\right)$ : Helimagnet, A-phase

D. $O\left(g_{\mathrm{so}}^{2}\right)$ : Helimagnet, NFL region

E. $O\left(g_{\mathrm{so}}^{4}\right)$ : Crystal-field effects

1. Pinning of the helix

2. The alignment transition, and the critical field $H_{\mathrm{c} 1}$

3. The polarization transition, and the critical field $H_{\mathrm{c} 1}{ }^{\prime}$

4. Pinning of the A-crystal and the NFL region

F. Beyond classical mean-field theory

III. Goldstone modes

A. $O\left(g_{\mathrm{so}}^{0}\right)$ : Ferromagnons

B. $O\left(g_{\mathrm{so}}^{2}\right)$ : Helimagnons

1. Symmetry arguments

2. Model calculation

C. $O\left(g_{\mathrm{so}}^{2}\right)$ : Skyrmionic Goldstone modes in the

A-phase and the NFL region

D. $O\left(g_{\mathrm{so}}^{4}\right)$ : Effects of the crystal-field terms
1. Symmetry arguments

2. Model calculation

3. Generalized helimagnons

4. A-phase, and NFL region

E. Summary of Goldstone modes, and temperature regimes

IV. Effects of Goldstone modes on electronic properties

A. Specific heat

1. Generalized helimagnons 14

2. A-phase

B. Relaxation times and transport coefficients

1. Generalized helimagnons

2. A-phase

C. Systems with quenched disorder

1. Generalized helimagnons

2. A-phase

3. NFL region

V. Summary, and Conclusion

16

Acknowledgments

17

A. The perpendicular conical state 18

B. Gaussian fluctuations in the aligned conical phase

C. Gaussian fluctuations in the pinned helical phase

D. Relaxation rates in ferromagnets 


\section{References}

\section{INTRODUCTION}

\section{A. Dzyaloshinsky-Moriya magnets}

Helical magnets are systems in which long-range magnetic order takes the form of a helix or spiral, such that in any given plane perpendicular to a preferred direction there is ferromagnetic order, but the direction of the magnetization rotates as one goes along the preferred axis. The pitch vector $\boldsymbol{q}$ of the helix points in the preferred direction, and its modulus $q \equiv|\boldsymbol{q}|$ is the pitch wave number, with $2 \pi / q$ equal to the helical wavelength. One mechanism for stabilizing this type of order over the homogeneous ferromagnet was pointed out by Dzyaloshinksky $\stackrel{1}{=}$ and Moriya. $\stackrel{2}{-}$ It relies on the spin-orbit interaction, which can lead, for certain lattice structures, to a term of the form $\boldsymbol{M} \cdot(\boldsymbol{\nabla} \times \boldsymbol{M})$ in the Hamiltonian or action, with $\boldsymbol{M}$ the magnetic order parameter. The presence of such a chiral term implies that a homogeneously magnetized state can always gain energy by means of a nonzero curl of the magnetization, which leads to a helical ground state. In a rotationally invariant system the direction of the pitch vector would be arbitrary (analogous to the arbitrary direction of the magnetization in an isotropic ferromagnet). However, the underlying lattice leads, via crystal-field effects, to a pinning of the helix in certain directions determined by the lattice. The crystal-field effects are due to the spin-orbit interaction, as is the Dzyaloshinsky-Moriya (DM) mechanism for helical order itself. Since the spin-orbit interaction is weak on an atomic or microscopic scale, with a dimensionless coupling constant $g_{\text {so }} \ll 1$, this leads to a hierarchy of energy or length scales that can be classified according to their dependence on powers of $g_{\text {so }}$. Finally, an external magnetic field provides an additional energy scale, and couples to the helix via an induced homogeneous component of the magnetization. This leads to a rich phase diagram that is the topic of the present paper.

\section{The phase diagram of $M n S i$}

A very well-studied helimagnet of DM-type is the metallic system $\mathrm{MnSi}$, which we will concentrate on; a very similar, but less extensively studied material is $\mathrm{FeGe}^{3}, \frac{3}{2}$ In zero magnetic field, and at ambient pressure, $\mathrm{MnSi}$ displays long-ranged helical order with $2 \pi / q \approx$ $180 \AA$ below a temperature $T_{\mathrm{c}} \approx 30 \mathrm{~K} \stackrel{4}{=} \mathrm{MnSi}$ crystallizes in the cubic B20 structure, and the helix is observed to be pinned in the $\langle 1,1,1\rangle$-directions $\frac{5,6}{.6}$ The corresponding space group is $\mathrm{P} 2{ }_{1} 3{ }^{\underline{7}}$ In a magnetic field $H$, the helix is superimposed by a homogeneous component of the magnetization, which leads to the so-called conical phase $\underline{\underline{6}} \mathrm{~A}$ magnetic field in the $[0,0,1]$-direction tilts the helix away from $\langle 1,1,1\rangle$ until the $\boldsymbol{q}$-vector aligns with the magnetic

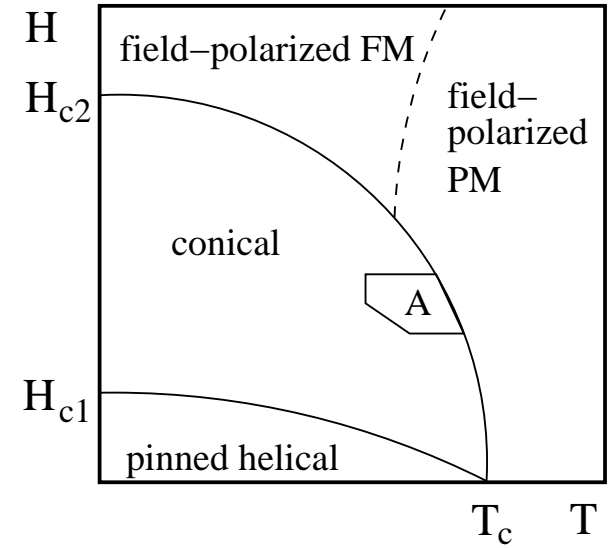

FIG. 1: Schematic phase diagram of MnSi in the $H-T$ plane showing the helical, conical, and A-phases, as well as the fieldpolarized ferromagnetic (FM) and paramagnetic (PM) states. See the text for a description of these phases.

field at a critical field strength $H_{\mathrm{c} 1}$. With increasing $H$ the amplitude of the helix decreases, and finally vanishes at a critical field $H_{\mathrm{c} 2}$, where the system enters a fieldpolarized ferromagnetic phase. Inside the conical phase at intermediate fields near $T_{\mathrm{c}}$ there is a region known as the A-phase. $\stackrel{8}{*}$ This was thought to represent a helix whose pitch vector is perpendicular to the magnetic field, $, 9,10$ but recently has been interpreted as a topological phase where three helices with co-planar $\boldsymbol{q}$-vectors form a skyrmion-like structure $\stackrel{11}{\underline{1}}$ The schematic phase diagram in the $H-T$-plane as observed experimentally is shown in Fig. 1. In addition to the phases shown, a possible second transition and spin-liquid phase at $H=0$ just above $T_{\mathrm{c}}$ has been reported recently $\underline{\underline{12}}$

Another interesting aspect of $\mathrm{MnSi}$ is its sensitivity to hydrostatic pressure. With increasing pressure $p$, the magnetic transition temperature $T_{\mathrm{c}}$ decreases until it vanishes at $p=p_{\mathrm{c}} \approx 15 \mathrm{kbar} \stackrel{13}{\underline{13}}$ The transition is second order or very weakly first order above a temperature of approximately $10 \mathrm{~K}$, and strongly first order at lower temperatures, with a tricritical point separating the two regimes. These features have been explained as universal properties of quantum ferromagnets in an approximation that neglects the helical order at longer length scales $\frac{14}{1}$ In the paramagnetic region at pressures up to approximately $2 p_{\mathrm{c}}$ and at temperatures $T \lesssim 10 \mathrm{~K}$, strong non-Fermi-liquid transport properties are observed, with the electrical resistivity $\rho_{\text {el }}$ displaying a temperature dependence $\rho_{\mathrm{el}} \propto T^{3 / 2}$ over almost three decades in temperature. 15 The origin of this behavior has recently been proposed to be a combination of columnar spin textures and very weak quenched disorder $\frac{16}{2}$ In a smaller region, above $p_{\mathrm{c}}$ and below a pressure-dependent temperature $T_{0}$, short-range (spin-liquid) helical order has been observed 17.18 and various explanations in terms of analogs of blue phases in liquid crystals have been proposed $\stackrel{19-22}{2}$ Recently, an anomalous Hall effect has 


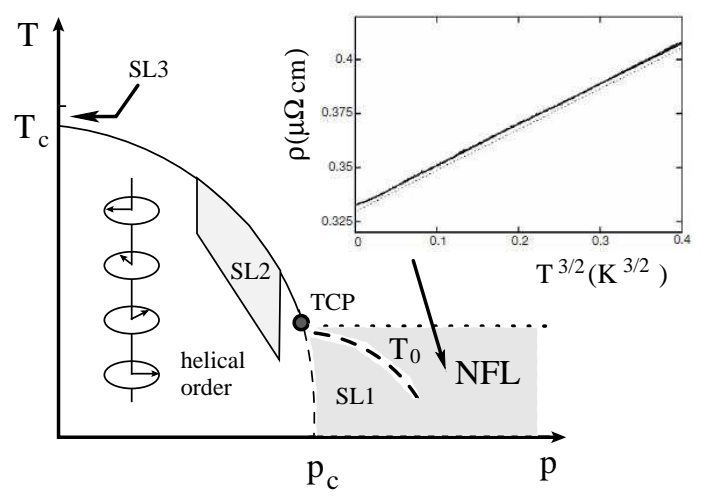

FIG. 2: Schematic phase diagram of MnSi in the T-p plane. The tricritical point (TCP) separates a line of second order transitions (solid line) from a line of first-order transitions (dashed line). The inset shows the resistivity data from Ref. 15 in the non-Fermi-liquid (NFL) region. The boundary of the NFL region (dotted line) is not sharp. SL1, SL2, and SL3 refer to the possible spin-liquid phases or regions reported in Refs. 17 and 18, 23, and 12, respectively.

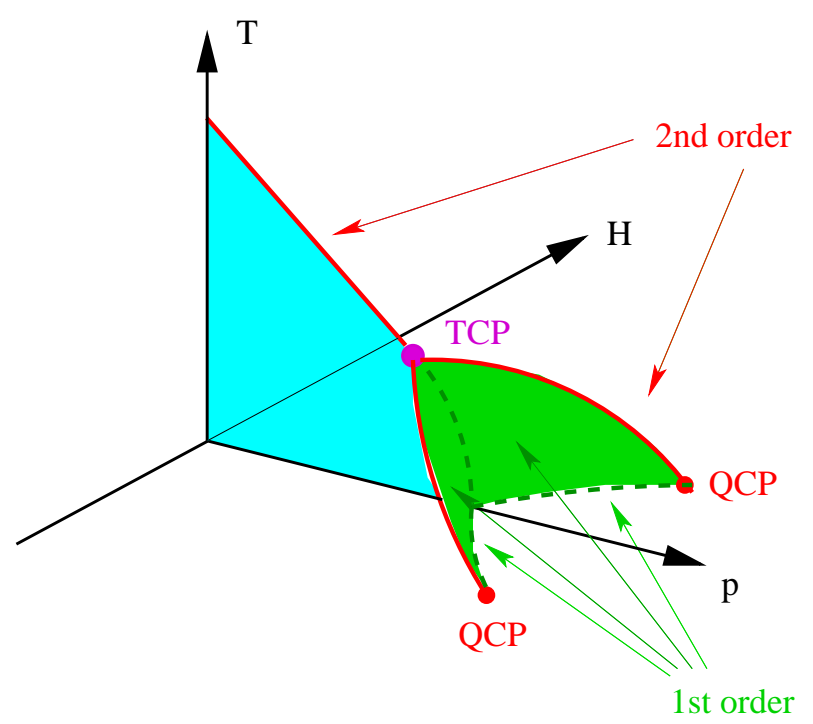

FIG. 3: Schematic phase diagram of $\mathrm{MnSi}$ in the space spanned by $T, p$, and $H$ showing the tricritical wings and quantum critical points (QCP).

been observed at intermediate pressures and below $T_{\mathrm{c}}$ in the helically ordered phase, and it has been suggested that this feature is related to the short-ranged order observed above $p_{\mathrm{c}} \stackrel{23}{\underline{2}}$ The phase diagram in the $T-p$ plane is shown in Fig. 2. If a magnetic field is applied in the vicinity of $p_{c}$, tricritical wings, i.e., surfaces of first-order transitions, emerge from the tricritical point that are believed to end in a pair of quantum critical points in the $T=0$ plane $\stackrel{15}{2}$ This feature, which is depicted in Fig. 3 , has been explained theoretically in Ref. 24 .

\section{Properties of the helical phase}

If one ignores crystal-field effects, i.e., in an isotropic model, the long-range helical order in the helical phase ( $\left.H=0, T<T_{\mathrm{c}}, p<p_{\mathrm{c}}\right)$ breaks the translational symmetry. This spontaneous breaking of a continuous symmetry leads to a Goldstone mode, the helimagnon. 25 The dispersion relation is anisotropic, and given by

$$
\omega_{\mathrm{HM}}(\boldsymbol{k})=\sqrt{c_{\| \mid} k_{\|}^{2}+c_{\perp} \boldsymbol{k}_{\perp}^{4}} .
$$

Here the wave vector $\boldsymbol{k}=\left(k_{\|}, \boldsymbol{k}_{\perp}\right)$ has been decomposed into components parallel and perpendicular, respectively, to the helical pitch vector $\boldsymbol{q}$, and $c_{\|}$and $c_{\perp}$ are elastic constants. The absence of a term proportional to $\boldsymbol{k}_{\perp}^{2}$ under the square root in Eq. (1.1) reflects rotational symmetry and is analogous to the nature of the Goldstone mode in either smectic or cholesteric liquid crystals $\underline{26}$ Crystal-field effects that couple the electrons to the underlying lattice break the rotational invariance and lead to a $\boldsymbol{k}_{\perp}^{2}$ term with a small prefactor, i.e., the dispersion relation becomes phonon-like. Both the single-particle relaxation rate $1 / \tau$ that results from the scattering of electronic quasi-particles by helimagnons and the thermal resistivity $\rho_{\text {th }}$ have a non-Fermi-liquid temperature dependence $1 / \tau \propto \rho_{\text {th }} \propto T^{3 / 2}$. In contrast, the transport relaxation rate that determines the electrical conductivity $\rho_{\mathrm{el}}$ in a Boltzmann approximation has a temperature dependence $1 / \tau_{\mathrm{el}}^{\mathrm{tr}} \propto \rho_{\mathrm{el}} \propto T^{5 / 2} \stackrel{27}{2}$ The properties of the helical phase in the presence of weak quenched disorder have also been investigated 28.29

In the present paper we will discuss the derivation of the phase diagram sketched above, as well as some refinements, within the context of a mean-field theory and with an emphasis on the $H-T$ plane. We will also determine the nature of the Goldstone modes, and their consequences for electronic properties, in various observed or proposed phases with long-range order. In addition to the specific heat, the single-particle relaxation rate, and the electrical conductivity we will consider the thermal resistivity $\rho_{\text {th }}$, whose temperature dependence is the same as that of the single-particle relaxation rate.

\section{B. Model, and energy scales}

\section{LGW functional}

We will consider a Landau-Ginzburg-Wilson (LGW) functional for a three-dimensional order parameter (OP) field $\boldsymbol{M}=\left(M_{1}, M_{2}, M_{3}\right)$ whose expectation value is proportional to the magnetization. We will consider an action that is appropriate for the helical magnets $\mathrm{MnSi}$ and FeGe, which crystallize in the cubic B20 structure with space group $\mathrm{P} 2{ }_{1} 3$. We will organize the action according to the dependence of its various constituents on powers of the spin-orbit interaction $g_{\text {so }}$. Within this scheme, we 
write

$$
\mathcal{A}=\mathcal{A}_{\mathrm{H}}+\mathcal{A}_{\mathrm{DM}}+\mathcal{A}_{\mathrm{cf}},
$$

where

$$
\begin{aligned}
\mathcal{A}_{\mathrm{H}}=\int_{V} d \boldsymbol{x} & {\left[\frac{t}{2} \boldsymbol{M}^{2}(\boldsymbol{x})+\frac{a}{2}(\nabla \boldsymbol{M}(\boldsymbol{x}))^{2}\right.} \\
+ & \frac{d}{2}(\nabla \cdot \boldsymbol{M}(\boldsymbol{x}))^{2}+\frac{u}{4}\left(\boldsymbol{M}^{2}(\boldsymbol{x})\right)^{2} \\
+ & \left.\frac{w}{4}\left(\nabla \boldsymbol{M}^{2}(\boldsymbol{x})\right)^{2}-\boldsymbol{H} \cdot \boldsymbol{M}(\boldsymbol{x})\right]
\end{aligned}
$$

is the action for an isotropic classical Heisenberg ferromagnet in a homogeneous external magnetic field $\boldsymbol{H}$. $\int_{V} d \boldsymbol{x}$ denotes a real-space integral over the system volume. $(\nabla \boldsymbol{M})^{2}$ stands for $\sum_{i, j=1}^{3} \partial_{i} M_{j} \partial^{i} M^{j}$, with $\partial_{i} \equiv \partial / \partial x_{i}$ the components of the gradient operator $\boldsymbol{\nabla} \equiv\left(\partial_{1}, \partial_{2}, \partial_{3}\right) \equiv\left(\partial_{x}, \partial_{y}, \partial_{z}\right)$. $t, a, d, u$, and $w$ are the parameters of the Landau theory and are related to the microscopic energy and length scales (see Sec. IB 2 below); they are of zeroth order in the spin-orbit coupling $g_{\text {so }}$. Equation (1.3) contains all analytic terms invariant under co-rotations of real space and OP space up to quartic order in $\boldsymbol{M}$ and bi-quadratic order in $\boldsymbol{M}$ and $\boldsymbol{\nabla} .30$ We have added one higher-order term, with coupling constant $w$, as an example of a class of terms that can stabilize unusual phases in helimagnets, although they are not of qualitative importance in ferromagnets. We will consider this term only in Appendix A

$$
\mathcal{A}_{\mathrm{DM}}=\frac{c}{2} \int_{V} d \boldsymbol{x} \boldsymbol{M}(\boldsymbol{x}) \cdot(\boldsymbol{\nabla} \times \boldsymbol{M}(\boldsymbol{x}))
$$

is the chiral Dzyaloshinksy-Moriya term that favors a nonvanishing curl of the magnetization. The existence of this term hinges on the spin-orbit coupling, as well as on the system not being invariant with respect to spatial inversion (due to the linear dependence on the gradient operator). The coupling constant $c$ is linear in $g_{\text {so }}$, and on dimensional grounds we have

$$
c=a k_{\mathrm{F}} g_{\mathrm{so}},
$$

with $k_{\mathrm{F}}$ the Fermi wave number which serves as the microscopic inverse length scale. In this context, this can be considered the definition of $g_{\text {so }}$.

The preceding contributions to the action are all invariant under either separate rotations, or co-rotations, in spin (or magnetization) space and real space. The spinorbit interaction couples the electron spin, and hence the magnetization, to the underlying lattice. Therefore, in addition to the rotationally invariant terms, any term that is invariant under elements of the space group connected with the crystal lattice is allowed. For the B20 structure of $\mathrm{MnSi}$ and FeGe, the appropriate space group is $\mathrm{P} 2{ }_{1} 3$. To quartic order in $\boldsymbol{M}$, and bi-quadratic order in $\boldsymbol{\nabla}$ and $\boldsymbol{M}$, the allowed terms in the action are the crystal-field terms

$$
\begin{array}{r}
\mathcal{A}_{\mathrm{cf}}=\int_{V} d \boldsymbol{x} \sum_{i=1}^{3}\left[\frac{b}{2}\left(\partial_{i} M_{i}(\boldsymbol{x})\right)^{2}+\frac{b_{1}}{2}\left(\partial_{i} M_{i+1}(\boldsymbol{x})\right)^{2}\right. \\
\left.+\frac{v}{4} M_{i}^{4}(\boldsymbol{x})\right]
\end{array}
$$

where $M_{4} \equiv M_{1}$. The last term is the usual cubic anisotropy that is always present in a magnet on a cubic lattice, and

$$
v=u^{\prime} g_{\mathrm{so}}^{4}
$$

with $\left|u^{\prime}\right| \approx u$. Of the gradient-squared terms, the first one also has cubic symmetry; the second one does not, but is invariant under elements of $\mathrm{P} 2_{1} 3$. On dimensional grounds, we have

$$
\begin{aligned}
b & =a^{\prime} g_{\mathrm{so}}^{2}, \\
b_{1} & =a_{1}^{\prime} g_{\mathrm{so}}^{2},
\end{aligned}
$$

with $\left|a^{\prime}\right| \approx\left|a_{1}^{\prime}\right| \approx a$

\section{Length and energy scales}

The various contributions to the action $\mathcal{A}$, and their dependencies on $g_{\text {so }}$, imply a hierarchy of energy scales and corresponding wave number or length scales. At zeroth order in $g_{\text {so }}$, we have the microscopic scale, which is represented by the Fermi energy $\epsilon_{\mathrm{F}}$ and the Fermi wave number $k_{\mathrm{F}}$. Fluctuations renormalize this to the critical scale, which is represented by the magnetic ordering temperature $T_{\mathrm{c}}$ and the corresponding length scale. The physics at these scales is described by $\mathcal{A}_{\mathrm{H}}$, Eq. (1.3).

The chiral DM term is balanced by the rotationally invariant gradient squared term in Eq. (1.3) that makes magnetization gradients energetically costly. As a result, the relevant gradient or momentum scale is of $O\left(g_{\mathrm{so}}\right)$, and hence the chiral wave number scale is given by the microscopic scale times $g_{\text {so }}$. This determines the parameters of the helix, in particular the helical pitch wave number $q \propto g_{\text {so }}$. In $\mathrm{MnSi}$ and FeGe, this wave number scale is on the order of 100 times smaller than the microscopic scale. $\mathcal{A}_{\mathrm{DM}}$ contains one explicit factor of $g_{\mathrm{so}}$ and one gradient, and hence its contribution to the free energy is of $O\left(g_{\text {so }}^{2}\right)$. The physics at this scale is described by $\mathcal{A}_{\mathrm{DM}}$ in conjunction with $\mathcal{A}_{\mathrm{H}}$.

At fourth order in $g_{\text {so }}$, crystal-field effects come into play. They pin the helix, are small compared to the chiral energy scale by another factor of $g_{\mathrm{so}}^{2}$, and are described by $\mathcal{A}_{\mathrm{cf}}$, Eqs. (1.5). Since gradients are effectively of $O\left(g_{\mathrm{so}}\right)$, see above, the contributions of all three terms in $\mathcal{A}_{\mathrm{cf}}$ to the free energy are of $O\left(g_{\text {so }}^{4}\right)$.

Finally, the external magnetic field sets a scale that is continuously tunable. 


\section{PHASE DIAGRAM}

We now derive the mean-field phase diagram for systems described by the action given in Eqs. (1.2) - (1.5). We will use the hierarchy of energy scales explained in Sec. IB to show how a more and more sophisticated phase diagram emerges as one keeps effects of higher and higher order in $g_{\text {so }}$.

To do so, we consider field configurations of the following form:

$$
\boldsymbol{M}(\boldsymbol{x})=\boldsymbol{m}_{0}+m_{1}^{+} \hat{\boldsymbol{e}}_{+} \cos (\boldsymbol{q} \cdot \boldsymbol{x})+m_{1}^{-} \hat{\boldsymbol{e}}_{-} \sin (\boldsymbol{q} \cdot \boldsymbol{x}) .
$$

Here $\boldsymbol{m}_{0}$ is a homogeneous component of the magnetization, $m_{1}^{ \pm}$are amplitudes of Fourier components with wave vector $\boldsymbol{q}$, and $\hat{\boldsymbol{e}}_{ \pm}$are two unit vectors that form a right-handed dreibein together with $\boldsymbol{q}$ :

$$
\hat{\boldsymbol{e}}_{+} \times \hat{\boldsymbol{e}}_{-}=\hat{\boldsymbol{q}} \quad, \quad \hat{\boldsymbol{q}} \times \hat{\boldsymbol{e}}_{+}=\hat{\boldsymbol{e}}_{-} \quad, \quad \hat{\boldsymbol{e}}_{-} \times \hat{\boldsymbol{q}}=\hat{\boldsymbol{e}}_{+},
$$

where $\hat{\boldsymbol{q}}=\boldsymbol{q} / q$. The sinusoidal terms in Eq. (2.1a) describe a helix with pitch vector $\boldsymbol{q}$. The helix is in general elliptically polarized, and it is useful to define a polarization parameter

$$
\pi=m_{1}^{-} / m_{1}^{+}
$$

Special cases are circular polarization, $\pi=1$, and linear polarization, $\pi=0$ or $\pi=\infty$. The motivation for the ansatz Eqs. (2.1) is provided by the fact that it gives the functional form of the global minimum of the action $\mathcal{A}$, Eqs. (1.2) - (1.5), if one neglects the crystal-field terms $\mathcal{A}_{\mathrm{cf}}$; that is, for the action up to $O\left(g_{\mathrm{so}}^{2}\right)$, see Ref. 11 and Sec. II B below.

\section{A. $O\left(g_{\text {so }}^{0}\right):$ Ferromagnet}

To zeroth order in $g_{\text {so }}$ the system is approximated by a ferromagnet. According to the action $\mathcal{A}_{\mathrm{H}}$, Eq. (1.3), for $H=0$ there is a second-order phase transition which in mean-field approximation occurs at $t=0$. For $H \neq 0$ there is a crossover at $t=0$ from a fieldpolarized paramagnetic state, where the magnetization extrapolates to zero for $H \rightarrow 0$, to a field-polarized ferromagnetic state where the magnetization extrapolates to $\boldsymbol{m}_{0}=\sqrt{-t / u} \hat{\boldsymbol{H}}$. The free energy density in mean-field approximation, $f=\mathcal{A} / V$, in a zero field is

$$
f=-t^{2} / 4 u \text {. }
$$

In a nonzero field, one has

$$
f=\frac{t}{2} m_{0}^{2}+\frac{u}{4} m_{0}^{4}-H m_{0},
$$

where $m_{0}$ is the solution of the mean-field equation of state

$$
t m_{0}+u m_{0}^{3}=H
$$

This is just a classical Heisenberg model, so it cannot explain the tricritical point and the associated tricritical wings. The latter emerge within a renormalized meanfield theory that takes into account the coupling of the magnetization to other electronic degrees of freedom. ${ }^{14}$ This leads to a fluctuation-induced first-order transition in analogy to the case of the nematic-to-smectic-A transition in liquid crystals,, 31 as well as to the tricritical wings and the quantum-critical points in an external magnetic field. ${ }^{24}$ The ferromagnetic approximation suffices for understanding the gross features of the phase diagram in the $T$ - $p$ plane.

\section{B. $O\left(g_{\text {so }}^{2}\right)$ : Helimagnet, conical phase}

To second order in $g_{\text {so }}$ we need to add the DM term, Eq. (1.4a), to the action. It is obvious that this term favors a nonzero curl of the magnetization, with the direction of the curl depending on the sign of $c$. However, the spatial variation of $\boldsymbol{M}$ will be limited by the other gradient terms in the action, the $(\nabla \boldsymbol{M})^{2}$ term in particular. We thus expect a spatial modulation of $\boldsymbol{M}$ on a length scale on the order of $a / c$. It is easy to check that the ansatz, Eqs. (2.1), with $\pi=1$, i.e., $m_{1}^{-}=m_{1}^{+} \equiv m_{1}$, and

$$
\begin{aligned}
\boldsymbol{q} & =q \hat{\boldsymbol{H}} \\
\boldsymbol{m}_{0} & =m_{0} \hat{\boldsymbol{H}} \\
m_{0} & =H /\left(c q-a q^{2}\right) \\
m_{1}^{2} & =\frac{1}{u}\left(-t+c q-a q^{2}-\frac{H^{2}}{\left(c q-a q^{2}\right)^{2}}\right)
\end{aligned}
$$

solves the saddle-point equations for the action $\mathcal{A}_{\mathrm{H}}+$ $\mathcal{A}_{\mathrm{DM}}$. In order to determine $q$, we extremize the resulting free energy with respect to $q . q=c / 2 a$ is a solution for all values of $H$. Finally, we need to ascertain that the solution is a minimum, which turns out to be the case for $t<a q^{2}$ and $H<a q^{2} \sqrt{-\left(t-a q^{2}\right) / u}$. We thus find that the field configuration

$$
\boldsymbol{M}(\boldsymbol{x})=m_{0} \hat{\boldsymbol{H}}+m_{1}\left[\hat{\boldsymbol{e}}_{+} \cos (q \hat{\boldsymbol{H}} \cdot \boldsymbol{x})+\hat{\boldsymbol{e}}_{-} \sin (q \hat{\boldsymbol{H}} \cdot \hat{\boldsymbol{x}})\right]
$$

with $\hat{\boldsymbol{e}}_{1}, \hat{\boldsymbol{e}}_{2}, \hat{\boldsymbol{H}}$ forming a dreibein, and

$$
\begin{aligned}
q & =c / 2 a \\
m_{0} & =H / a q^{2} \\
m_{1} & =\sqrt{-r / u} \sqrt{1-\left(H / H_{\mathrm{c} 2}\right)^{2}}
\end{aligned}
$$

where

$$
\begin{aligned}
r & =t-a q^{2} \\
H_{\mathrm{c} 2} & =a q^{2} \sqrt{-r / u}
\end{aligned}
$$

minimizes the free energy in the parameter range $r<0$, $H<H_{\mathrm{c} 2}$. We will refer to this state as the aligned conical state (ACS), to distinguish it from the perpendicular 
conical state discussed in Appendix A. The ACS is actually a global minimum, as can be seen by writing the action as a sum of positive semi-definite terms that are individually minimized by this state $\underline{\underline{11}}$ The mean-field free energy density in that range is

$$
f=\frac{-1}{2}\left(\frac{r^{2}}{2 u}+\frac{H^{2}}{a q^{2}}\right) .
$$

Equations (2.6) describe the helical phase for $H=0$ and the conical phase for $0<H<H_{\mathrm{c} 2}$. Comparing Eqs. (2.3) and (2.8) we see that the helical transition preempts the ferromagnetic one.

For $H \rightarrow H_{\mathrm{c} 2}$ from below the helical component of the magnetization vanishes, and the free energy, Eq. (2.8), approaches that of the ferromagnet, Eqs. (2.4). For $H>$ $H_{\mathrm{c} 2}$ the equation of state and the free energy for the DM action $\mathcal{A}_{\mathrm{H}}+\mathcal{A}_{\mathrm{DM}}$ are the same as for a ferromagnet with action $\mathcal{A}_{\mathrm{H}}$.

These considerations account for the structure of the phase diagram shown in Fig. 1 except for the field $H_{\mathrm{c} 1}$ and the A-phase.

\section{C. $O\left(g_{\text {so }}^{2}\right)$ : Helimagnet, A-phase}

Recent neutron-scattering experiments by Mühlbauer et al. 11 are consistent with the notion that the A-phase is characterized by spin textures that form line defects in the direction of the magnetic field, with the lines forming a hexagonal lattice, the A-crystal, in the plane perpendicular to the field. This experimental observation led the authors of Ref. 11 to suggest a skyrmion state consisting of three co-planar helices as underlying the A-phase:

$$
\begin{gathered}
\boldsymbol{M}(\boldsymbol{x})=m_{0} \hat{\boldsymbol{H}} \\
+m_{1} \sum_{i=1}^{3}\left[\hat{\boldsymbol{e}}_{+}^{(i)} \cos \left(\boldsymbol{q}^{(i)} \cdot \boldsymbol{x}\right)+\hat{\boldsymbol{e}}_{-}^{(i)} \sin \left(\boldsymbol{q}^{(i)} \cdot \boldsymbol{x}\right)\right],
\end{gathered}
$$

where the pitch vectors $\boldsymbol{q}^{(i)}$ all have the same modulus $q$, are perpendicular to $\boldsymbol{H}$, the angle between adjacent pitch vectors is $2 \pi / 3$, and $\hat{\boldsymbol{e}}_{+}^{(i)}, \hat{\boldsymbol{e}}_{-}^{(i)}$, and $\boldsymbol{q}^{(i)}$ form a righthanded dreibein for each value of $i$. The values of $q, m_{0}$, and $m_{1}$ are obtained by minimizing the action.

The free energy difference between this state and the conical one has a minimum at $H \approx 0.4 H_{\mathrm{c} 2}$, but it is still positive even at the minimum. However, Ref. 11 found that taking into account Gaussian fluctuations stabilizes the state with respect to the conical one. It should be noted that Eq. (2.9) is not a solution of the saddle-point equations for the action given by Eqs. (1.2)(1.5), and therefore cannot be a true local minimum of the free energy. Also, the relation between this ansatz and what are commonly called skyrmionic spin configurations, which are solutions of the saddle-point equations (see Ref. 21 and references therein), is not clear. Most likely it represents a single-Fourier-component approximation to a saddle point and is a Bloch-type description

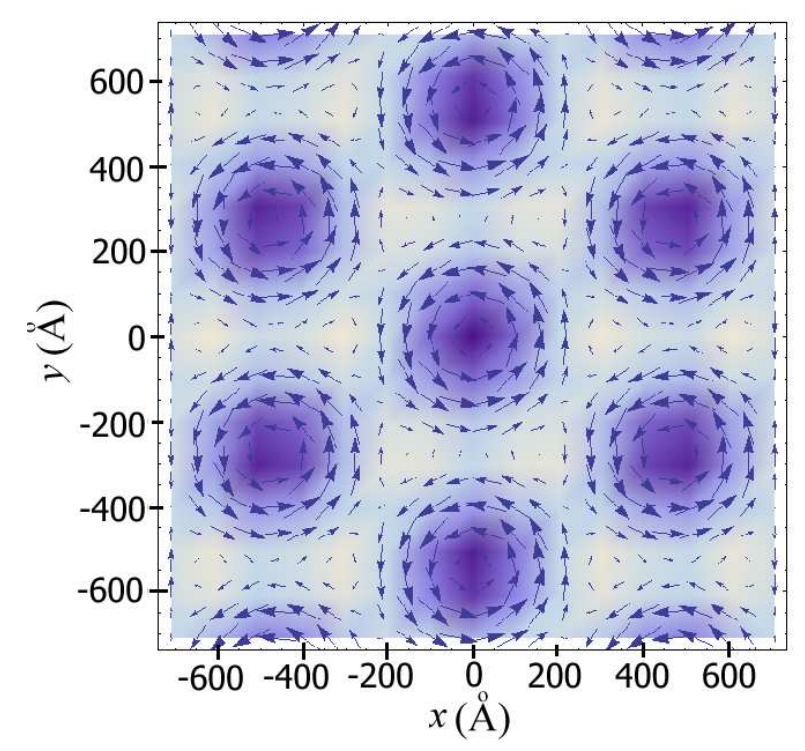

FIG. 4: Spin configuration as given by Eq. (2.9) with the $\boldsymbol{q}$ vectors in the $x-y$ plane, $q=0.0133 \AA^{-1}, m_{0}=0.0146$, and $m_{1}=0.0323$. The arrows represent projections of the spins into the plane. The dark and light regions denote spin directions antiparallel and parallel to $\hat{z}$, respectively.

of skyrmions as opposed to a Wannier-type description of isolated skyrmions in Ref. 21. In any case, it describes a hexagonal array of line defects with the spin antiparallel to the magnetic field at the defect centers, and parallel at points on the cell boundaries, see Fig. 4, in qualitative agreement with the neutron scattering data.

Earlier, Grigoriev et al $\stackrel{10}{\underline{n}}$ had proposed a single-helix state with the pitch vector oriented perpendicular to the external field. Although current experimental evidence favors a skyrmion state as a candidate for the A-phase, it is still of interest to discuss such a perpendicular conical state (PCS), since it might be a viable candidate for the ground state in some other part of the phase diagram of helimagnets. We therefore briefly discuss the PCS and its properties in Appendix $\mathrm{A}$.

\section{D. $O\left(g_{\mathrm{so}}^{2}\right)$ : Helimagnet, NFL region}

The skyrmion lattice approximately described by Eq. (2.9) can melt, which will lead to a skyrmion liquid. In such a state the line defects shown in Fig. 4 still exist, but they no longer form a lattice. Rather, their fluctuations in the plane perpendicular to the line, which are illustrated in Fig. 5 , have become so large that the longrange order is destroyed. Such a state has recently been proposed to represent the NFL region shown in Fig. $2 \underline{16}^{16}$ Although it is not an ordered phase, such a state has much in common with the A-phase and we will discuss it in the context of the ordered phases. 


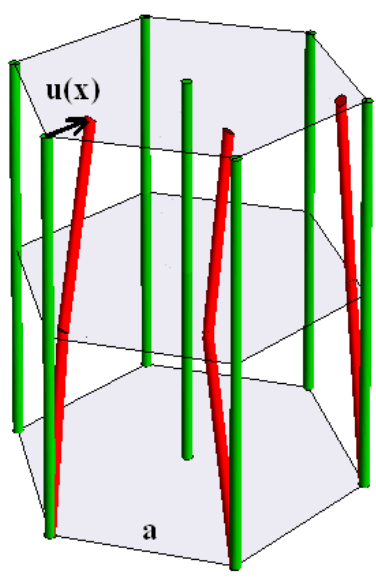

FIG. 5: Schematic rendering of the hexagonal lattice of skyrmion lines shown in Fig. 4 and of fluctuations about this state. $a$ is the lattice constant, and $\boldsymbol{u}(\boldsymbol{x})$ is a displacement vector.

\section{E. $O\left(g_{\text {so }}^{4}\right)$ : Crystal-field effects}

To fourth order in $g_{\text {so }}$, we need to take into account the crystal-field terms shown in Eq. (1.5a). This makes the saddle-point equations very complicated, and no exact solution is known. We therefore take a variational approach by inserting Eq. (2.1a) into the action and minimizing with respect to the parameters of the ansatz. Of all the members of the class of functions represented by Eqs. 2.1) this will yield the one with the lowest free energy.

By writing Eq. 1.5a we have fixed the coordinate system by choosing the crystallographic axes to be the $x, y, z \equiv 1,2,3$ axes. We thus are no longer free to choose the direction of $\boldsymbol{H}, \mathbf{m}_{0}$, or $\boldsymbol{q}$. For simplicity, we will consider only the case of a magnetic field along the $z$-axis: $\boldsymbol{H}=(0,0, H) . \quad \hat{\boldsymbol{q}} \equiv \boldsymbol{q} / q$ we parameterize in terms of angles $\vartheta$ and $\varphi$ as follows,

$$
\hat{\boldsymbol{q}}=(\sin \vartheta \cos \varphi, \sin \vartheta \sin \varphi, \cos \vartheta) \equiv\left(\beta_{1}, \beta_{2}, \beta_{3}\right)
$$

with $\sum_{i=1}^{3} \beta_{i}^{2}=1$. This leaves one free parameter for $\hat{\boldsymbol{e}}_{+}$, namely, an azimuthal angle $\varphi_{e}$ :

$$
\begin{aligned}
\hat{\boldsymbol{e}}_{+}= & \left(\cos \vartheta \cos \varphi, \sin \varphi_{e}-\sin \varphi \cos \varphi_{e},\right. \\
& \cos \vartheta \sin \varphi \sin \varphi_{e}+\cos \varphi \cos \varphi_{e}, \\
& \left.-\sin \vartheta \sin \varphi_{e}\right) .
\end{aligned}
$$

This uniquely determines $\hat{\boldsymbol{e}}_{-}=\hat{\boldsymbol{q}} \times \hat{\boldsymbol{e}}_{+}$. Finally, $\boldsymbol{m}_{0}$ in general needs to be decomposed into components parallel and perpendicular, respectively, to $\boldsymbol{q} \cdot \frac{32}{}$ However, while a perpendicular component can lead to a slightly lower free energy, it has no qualitative effects on the structure of the phase diagram, and we therefore restrict our ansatz to

$$
\boldsymbol{m}_{0}=m_{0} \hat{\boldsymbol{q}} .
$$

We further assume that the system is sufficiently close to a second order or weakly first order phase transition that one can neglect the last term in Eq. (1.5a). With these approximations, the free energy does not depend on the angle $\varphi_{e}$ and is completely parameterized in terms of six parameters, namely: two amplitudes, $m_{0}$ and $m_{1}=$ $\left[\left(m_{1}^{+}\right)^{2}+\left(m_{1}^{-}\right)^{2}\right]^{1 / 2} / \sqrt{2}$, the polarization parameter $\pi$, the modulus $q$, and the two direction angles $\vartheta$ and $\varphi$. We find

$$
\begin{aligned}
f= & \frac{1}{2} \delta t m_{0}^{2}+\frac{r}{4}\left(m_{0}^{2}+m_{1}^{2}\right)+\frac{u}{4}\left(m_{0}^{2}+m_{1}^{2}\right)^{2}+\frac{1}{2} m_{1}^{2}\left[\delta t+a q^{2}-c q+\frac{1}{2} c q(\delta \pi)^{2}\right]-H m_{0} \cos \vartheta \\
& +\frac{b}{4} m_{1}^{2} q^{2} B_{\mathrm{s}}(\vartheta, \varphi)+\frac{b_{1}}{4} m_{1}^{2} q^{2} B_{1 \mathrm{~s}}(\vartheta, \varphi)-\frac{b}{4} m_{1}^{2} q^{2} \delta \pi B_{\mathrm{a}}(\vartheta, \varphi)-\frac{b_{1}}{4} m_{1}^{2} q^{2} \delta \pi B_{1 \mathrm{a}}(\vartheta, \varphi)+O\left(g_{\mathrm{so}}^{8}\right) .
\end{aligned}
$$

Here we have defined $r=t-\delta t$, with $\delta t$ arbitrary at this point. We also have made use of the fact that we know, from Sec. IIB, that the physical solution has the property $\pi=1+\delta \pi$ with $\delta \pi=O\left(g_{\text {so }}^{2}\right)$, and have expanded in powers of $\delta \pi$. The angle-dependent functions in Eq. (2.11a) are

$$
\begin{aligned}
B_{\mathrm{s}, \mathrm{a}}(\vartheta, \varphi) & =B^{+}(\vartheta, \varphi) \pm B^{-}(\vartheta, \varphi) \\
B_{1 \mathrm{~s}, \mathrm{a}}(\vartheta, \varphi) & =B_{1}^{+}(\vartheta, \varphi) \pm B_{1}^{-}(\vartheta, \varphi)
\end{aligned}
$$

where

$$
\begin{aligned}
B^{+}(\vartheta, \varphi)= & 2 \sin ^{2} \vartheta \sin ^{2} \varphi \cos ^{2} \varphi \\
B^{-}(\vartheta, \varphi)= & \sin ^{2} \vartheta \cos ^{2} \vartheta\left(1+\sin ^{4} \varphi+\cos ^{4} \varphi\right) \\
B_{1}^{+}(\vartheta, \varphi)= & \sin ^{2} \vartheta \cos ^{4} \varphi+\cos ^{2} \vartheta \sin ^{2} \varphi \\
B_{1}^{-}(\vartheta, \varphi)= & \sin ^{4} \vartheta \sin ^{2} \varphi+\cos ^{4} \vartheta \cos ^{2} \varphi \\
& +\sin ^{2} \vartheta \cos ^{2} \vartheta \sin ^{2} \varphi \cos ^{2} \varphi
\end{aligned}
$$

We now need to minimize the free energy with respect to the six parameters. We will first consider the case $H=0$ to understand the pinning of the helix by the crystal-field terms, and then determined the effects of a magnetic field. Furthermore, in order to keep the discus- 
sion transparent we will initially restrict ourselves to an ansatz with a circular polarization, $\delta \pi=0$. This suffices to understand the existence of the critical field $H_{\mathrm{c} 1}$. We will then generalize the ansatz to allow for a non-circular polarization and show that this leads to a splitting of the transition at $H_{\mathrm{c} 1}$, with a first order transition from a circularly polarized helix to an elliptically polarized one at a critical field $H_{\mathrm{c} 1}{ }^{\prime}<H_{\mathrm{c} 1}$ preceding the alignment transition at $H_{\mathrm{c} 1}$.

\section{Pinning of the helix}

We consider the system at $H=0$ and initially restrict our ansatz to the case of circular polarization, $\delta \pi=0$. The remaining angular dependence in the free energy, Eq. (2.11a), is contained in the functions $B_{\mathrm{s}}$ and $B_{1 \mathrm{~s}}$. Minimizing with respect to $\varphi$ we find that there are two cases.

Case (1): $\varphi=0, \pi / 2, \pi, 3 \pi / 2$. This implies $\hat{\boldsymbol{q}}=$ $(0, \pm \sin \vartheta, \cos \vartheta)$ or $( \pm \sin \vartheta, 0, \cos \vartheta)$, and $B_{\mathrm{s}}=$ $\frac{1}{2} \sin ^{2} 2 \vartheta, B_{1 \mathrm{~s}}=\sin ^{2} \vartheta+\cos ^{4} \vartheta$. Minimizing with respect to $\vartheta$ leads to two subcases:

Case (1a): $2 b<b_{1}$. The free energy is minimized by $\vartheta=\pi / 4,3 \pi / 4,5 \pi / 4,7 \pi / 4$, which implies $\hat{\boldsymbol{q}}=(1,0,1) / \sqrt{2}$ or equivalent.

Case (1b): $2 b>b_{1}$. The free energy is minimized by $\vartheta=0, \pi / 2, \pi, 3 \pi / 2$, which implies $\hat{\boldsymbol{q}}=(1,0,0)$ or equivalent.

Case (2): $\varphi=\pi / 4,3 \pi / 4,5 \pi / 4,7 \pi / 4$. This implies $\hat{\boldsymbol{q}}=$ $(\sin \vartheta / \sqrt{2}, \sin \vartheta / \sqrt{2}, \cos \vartheta)$ or equivalent, and $B_{\mathrm{s}}=$ $1-\sin ^{4} \vartheta / 2-\cos ^{4} \vartheta, B_{1 \mathrm{~s}}=1-\sin ^{2} \vartheta+3 \sin ^{4} \vartheta / 4$. Minimizing with respect to $\vartheta$ yields

Case (2a): $2 b<b_{1}$. The free energy is minimized by $\vartheta= \pm \arcsin \sqrt{2 / 3}$, which implies $\hat{\boldsymbol{q}}=(1,1,1) / \sqrt{3}$ or equivalent.

Case (2b): $2 b>b_{1}$. The free energy is minimized by $\vartheta=0, \pi$, which implies $\hat{\boldsymbol{q}}=(0,0, \pm 1)$.

By comparing the resulting free energies for these cases we see that Case (1b) provides the minimum for $b>b_{1} / 2$, whereas Case (2a) provides the minimum for $b<b_{1} / 2$. This is a generalization of the result obtained in Ref. 33 , which considered a model with $b_{1}=0$.

In $\mathrm{MnSi}$, the pinning is observed to be in the $\langle 1,1,1\rangle$ directions, which implies $b<b_{1} / 2$, and we will focus on this case from now on. Minimizing the free energy with respect to $q$, we find

$$
q=\frac{c / 2}{a+\left(b+b_{1}\right) / 3}
$$

which generalizes Eq. 2.6b). Choosing $\delta t=c q / 2$ and minimizing with respect to $m_{1}$ we finally have

$$
f=-r^{2} / 4 u
$$

where

$$
r=t-\delta t
$$

with

$$
\delta t=\frac{c^{2} / 4}{a+\left(b+b_{1}\right) / 3} .
$$

These results are valid for $H=0$ and $b<b_{1} / 2$.

2. The alignment transition, and the critical field $H_{c 1}$

For $H>0$ we expect the pitch vector to move away from $(1,1,1)$ towards $(0,0,1)$. The calculation proceeds as for $H=0$, except that now the minimization with respect to $\vartheta$ yields an $H$-dependent result. For Case (2a) we find

$$
\beta_{3} \equiv \cos \vartheta= \begin{cases}1 & \text { if } H \geq H_{\mathrm{c} 1} \\ \frac{1}{3}\left(1+2 H^{2} / H_{\mathrm{c} 1}{ }^{2}\right) & \text { if } H<H_{\mathrm{c} 1}\end{cases}
$$

where (remember $r<0$ and $b<b_{1} / 2$ in the ordered phase for Case (2a))

$$
H_{\mathrm{c} 1}^{2}=r\left(b-b_{1} / 2\right)(\delta t)^{2} / u a .
$$

The helical pitch vector $\hat{\boldsymbol{q}}$ thus moves continuously along the shortest path from its initial value, $(1,1,1) / \sqrt{3}$ at $H=0$, to $(0,0,1)$ at $H=H_{\mathrm{c} 1}$, and remains in that position for $H>H_{\mathrm{c} 1}$. There thus is a second order transition at $H=H_{\mathrm{c} 1}{ }^{32}$ that we refer to as the alignment transition. An inspection shows that Case (1b) has a larger free energy for all $H<H_{\mathrm{c} 1}$.

For reference in the next subsection we mention that if one expands the free energy for small values of $\vartheta$, and looks for the instability of the solution with $\vartheta=0$, one finds that the latter occurs at $H=H_{\mathrm{c} 1}$, as expected.

\section{The polarization transition, and the critical field $H_{c 1}{ }^{\prime}$}

The circular polarization ansatz we have used so far explains the two critical fields $H_{\mathrm{c} 1}$ and $H_{\mathrm{c} 2}$ observed in $\mathrm{MnSi}$. However, the solution obtained in this way misses a qualitative feature, as was first pointed out in Ref. 34 on symmetry grounds. Since in general $b \neq b_{1}$, the crystalfield contribution to the action, Eq. (1.5a), is not invariant under $x \leftrightarrow y$. As a result, there is no reason for the $x$ and $y$-components of $\hat{\boldsymbol{q}}$ to become nonzero at the same value of $H$ as $H$ is lowered from above, yet the solution constructed in the previous subsection has this property. Clearly, this is a result of the fact that our ansatz with circular polarization possesses cubic symmetry, while the action does not. In general, one therefore expects two separate transitions in the vicinity of $H_{\mathrm{c} 1}$; one where the $x$-component of $\hat{\boldsymbol{q}}$ becomes nonzero, and a separate one where the $y$-component becomes nonzero. We now show 
that this expectation is borne out if we allow for a noncircular polarization of the helix, which breaks the cubic symmetry of the ansatz.

Consider the full Eq. (2.11a), allowing for $\delta \pi \neq 0$. Minimizing with respect to $\varphi$ we see that there are two distinct cases.

Case (1): $\varphi=0, \pi / 2, \pi, 3 \pi / 2$ and $\delta \pi$ arbitrary. Minimizing with respect to $\delta \pi$ yields

$$
\delta \pi(\vartheta)=\frac{q}{2 c}\left[b B_{\mathrm{a}}(\vartheta)+b_{1} B_{1 \mathrm{a}}(\vartheta)\right] .
$$

That is, the polarization is in general elliptical. We have $B^{+}=0, B^{-}=2 \sin ^{2} \vartheta \cos ^{2} \vartheta$, which leads to

$$
\begin{aligned}
B_{\mathrm{s}, \mathrm{a}} & = \pm \frac{1}{2} \sin ^{2} 2 \vartheta \\
B_{1 \mathrm{~s}} & =\sin ^{2} \vartheta+\cos ^{4} \vartheta .
\end{aligned}
$$

Considering $B_{1 \mathrm{a}}$ we find two subcases. The first one is

Case (1) (i): $\varphi=0, \pi$, which implies

$$
\hat{\boldsymbol{q}}=(0, \pm \sin \vartheta, \cos \vartheta)
$$

and

$$
B_{1 \mathrm{a}}=\sin ^{2} \vartheta-\cos ^{4} \vartheta
$$

The second one is

Case (1)(ii): $\varphi=\pi / 2,3 \pi / 2$, which implies

$$
\hat{\boldsymbol{q}}=( \pm \sin \vartheta, 0, \cos \vartheta),
$$

and

$$
B_{1 s, a}=\cos ^{2} \vartheta \pm \sin ^{4} \vartheta .
$$

The second case is

Case (2): $\varphi=\pi / 4,3 \pi / 4,5 \pi / 4,7 \pi / 4$ and

$$
\delta \pi=0
$$

That is, the polarization is circular. This implies

$$
\hat{\boldsymbol{q}}=\left(\frac{1}{\sqrt{2}} \sin \vartheta, \frac{1}{\sqrt{2}} \sin \vartheta, \cos \vartheta\right),
$$

and

$$
\begin{aligned}
B_{\mathrm{s}} & =1-\frac{1}{2} \sin ^{4} \vartheta-\cos ^{4} \vartheta \\
B_{1, \mathrm{~s}} & =1-\sin ^{2} \vartheta+\frac{3}{4} \sin ^{4} \vartheta
\end{aligned}
$$

Now first consider the case $H=0$. Minimizing with respect to $\vartheta$ one finds that, for $b<b_{1} / 2$, Case (2) yields the lower free energy 35 The physical solution is thus a circularly polarized helix pinned in the $\langle 1,1,1\rangle$ directions,

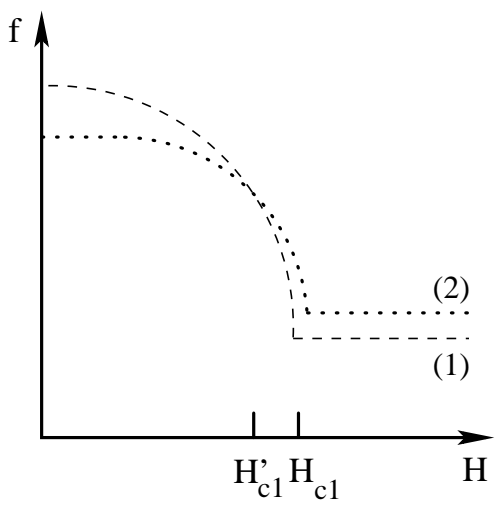

FIG. 6: Schematic plot of the free energy density as a function of $H$ for Case (1) (dashed line) and Case (2) (dotted line), respectively.

and the relaxation of the condition we had imposed in Sec. IIE 1 does not change anything. For $b>b_{1} / 2$, the physical solution is an elliptically polarized helix pinned in the $\langle 0,0,1\rangle$ directions.

Next we consider a solution with $\vartheta=0$, which we expect to be stable for sufficiently large $H$. It is easy to see that Case (1), which takes advantage of the possibility of an elliptical polarization, has a free energy that is lower than that of Case (2) by a term of $O\left(g_{\text {so }}^{6}\right)$ everywhere in the conical phase, i.e., for $H<H_{\mathrm{c} 2}$. We next look for the instability of the $\vartheta=0$ solution at small $H$, which can be found by expanding the action to second order in $\vartheta$ and looking for the zero of the coefficient of the quadratic term. As expected, this instability occurs at a field $H_{\mathrm{c} 1}=O\left(g_{\mathrm{so}}^{3}\right)$. To leading order in $g_{\mathrm{so}}, H_{\mathrm{c} 1}$ is given by Eq. (2.14b). The values of $H_{\mathrm{c} 1}$ for the two cases are different, but the difference is only of $O\left(g_{\mathrm{so}}^{5}\right)$ and is irrelevant for the following argument.

We now have the following situation. For $H<H_{\mathrm{c} 1}$, the free energy of Case (1) is lower by a term of $O\left(g_{\mathrm{so}}^{6}\right)$. However, we know that at $H=0$ the free energy of Case (2) is lower by a term of $O\left(g_{\mathrm{so}}^{4}\right)$. The two solutions cross at a field $H_{\mathrm{c} 1}{ }^{\prime}$ given by

$$
\frac{H_{\mathrm{c} 1}{ }^{\prime}}{H_{\mathrm{c} 1}}=1-\frac{1}{2} \sqrt{\frac{3 b_{1}^{2}}{4 a\left|b-b_{1} / 2\right|}}=1-O\left(g_{\mathrm{so}}\right),
$$

see Fig. 6. At this value of $H$, the state will change discontinuously from an elliptically polarized helix with a pitch vector given by either Eq. (2.16a) or (2.17a) to a circularly polarized one with a pitch vector given by Eq. (2.18b).

We now have the following progression of phases and phase transitions as the magnetic field is lowered from a value greater than $H_{\mathrm{c} 2}$ :

$H>H_{\mathrm{c} 2}$ : Field-polarized state, no helix.

$H=H_{\mathrm{c} 2} \propto g_{\mathrm{so}}^{2}$ : Second-order transition to a conical state with an elliptically polarized helix, $\hat{\boldsymbol{q}}=(0,0,1)$.

$H=H_{\mathrm{c} 1} \propto g_{\mathrm{so}}^{3}$ : Second-order transition to a conical state with elliptical polarization as above, but $\hat{\boldsymbol{q}}=$ 


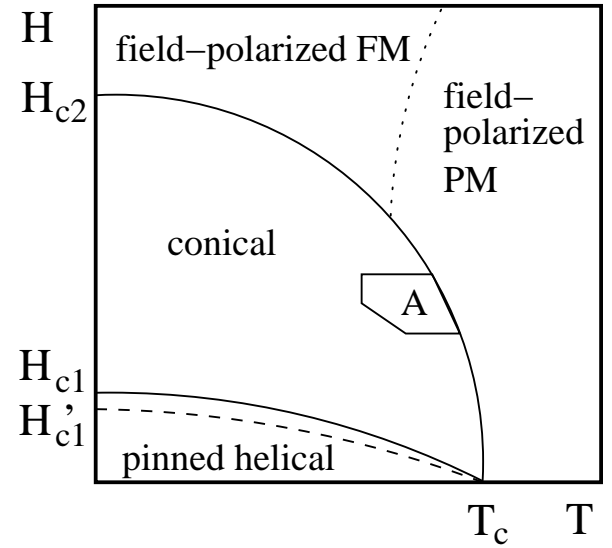

FIG. 7: Phase diagram of MnSi in the $H-T$ plane showing the predicted polarization transition at $H_{\mathrm{c} 1}{ }^{\prime}$ (first order, dashed line) in addition to the second-order transitions at $H_{\mathrm{c} 1}$ and $H_{\mathrm{c} 2}$. The nature of the transition from the conical phase to the A-phase, or the A-phase to the paramagnetic phase, has not been investigated so far. The dotted line denotes the crossover from a field-polarized paramagnet to a fieldpolarized ferromagnet.

$(0, \sin \vartheta, \cos \vartheta) . \vartheta$ increases from zero with decreasing $H$.

$H=H_{\mathrm{c} 1}{ }^{\prime}=H_{\mathrm{c} 1}\left[1-O\left(g_{\mathrm{so}}\right)\right]:$ First-order transition to a conical state with circular polarization and $\hat{\boldsymbol{q}}=$ $\left(\frac{1}{\sqrt{2}} \sin \vartheta, \frac{1}{\sqrt{2}} \sin \vartheta, \cos \vartheta\right) . \vartheta$ increases from its value at $H_{\mathrm{c} 1}{ }^{\prime}$ with decreasing $H$.

$H=0$ : System reaches helical state with circular polarization and $\hat{\boldsymbol{q}}=(1,1,1) / \sqrt{3}$.

The phase diagram is thus predicted to have the structure shown in Fig. 7, with the second order alignment transition at $H_{\mathrm{c} 1}$ followed by a first-order polarization (and re-alignment) transition at $H_{\mathrm{c} 1}{ }^{\prime}$. The latter has so far not been observed experimentally. It is an explicit realization of the type of transition first predicted by Walker ${ }^{34}$ on symmetry grounds. It needs to be stressed that the detailed features of this transition are restricted by our ansatz, Eqs. (2.1); the states obtained are not true minima of the action. However, the basic physical idea, which is expected to be realized in the true ground state as well, is as follows. In the conical phase, where the helical pitch vector is aligned with the magnetic field, the system can take advantage of the lack of cubic symmetry of the action, Eq. (1.5a), by forming a helix with a non-circular polarization. This leads to an energy gain, compared to a circularly polarized state, of order $g_{\mathrm{so}}^{6}$. However, it forces the pitch vector into either the $y=0$ or $x=0$ plane, i.e., Case (1) above. At low fields, these states have a free energy that is larger by a term of $O\left(g_{\mathrm{so}}^{4}\right)$ than the states with the pitch vector along a cubic diagonal, which requires a circular polarization. The competition between these two effects leads to the first order transition at $H_{\mathrm{c} 1}$, where both the polarization and the orientation of the pitch vector change discontinuously.

\section{Pinning of the A-crystal and the NFL region}

Experimentally, the A-crystal is found to be pinned very weakly, and theoretical considerations conclude that the pinning potential is only of $O\left(g_{\mathrm{so}}^{6}\right) \underline{\underline{11}}$ This is a consequence of the six-fold rotation symmetry of the A-crystal. Similarly one expects the (average) direction of the fluctuating defect lines in the NFL region to be pinned only very weakly.

\section{F. Beyond classical mean-field theory}

We have treated the phase transitions that we have discussed in this section within classical mean-field theory, and the question arises what changes will result from the consideration of fluctuations, classical or quantum. One example of the effects of fluctuations is the tricritical point and the associated tricritical wings shown in Fig. 3, which result from quantum fluctuations that can be taken into account within a renormalized meanfield theory. $\stackrel{14.31}{=}$ Elsewhere in the phase diagram, fluctuations are also of qualitative importance. Consider, for instance, the transition from the conical phase to the field-polarized phase at the critical field $H_{\mathrm{c} 2}$, see Fig. 1. The transition is characterized by the vanishing of the one-dimensional order parameter $m_{1}$, the amplitude of the helix, see Eqs. (2.6). The action is invariant under $m_{1} \rightarrow-m_{1}$, and we therefore expect this transition at nonzero temperature to be in the universality class of the classical Ising model ${ }^{36}$ At zero temperature the statics and the dynamics will couple,$\stackrel{37,38}{, 3}$ and one expects the quantum phase transition to be described by Hertz's mode ${ }^{38}$ with a scalar order parameter.

\section{GOLDSTONE MODES}

Goldstone's theorem states that if a continuous symmetry described by a Lie group $G$ is spontaneously broken, with the remaining subgroup in the brokensymmetry phase being $H$ (not to be confused with the magnetic field), then in the ordered phase there are $n$ soft or massless modes, with $n$ equal to the dimension-

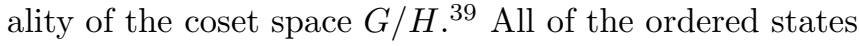
discussed in Sec. I break a continuous symmetry, and therefore there must be one or more soft modes in the ordered phase .40 The number and functional form of the Goldstone modes in the various phases can be determined from general arguments. In this section we will do so, and we will augment these arguments by explicit calculations in some cases where this is feasible. Via a coupling to the conduction electrons, the Goldstone modes have interesting consequences for various observables; this is the topic of Sec. IV. 


\section{A. $O\left(g_{\text {so }}^{0}\right)$ : Ferromagnons}

To zeroth order in $g_{\text {so }}$ the system is a ferromagnet, see Sec. II A The relevant symmetry is the rotation group, $G=O(3)$, which in the ordered state is spontaneously broken to $H=O(2)$. Hence there are $\operatorname{dim}(O(3) / O(2))=$ 2 Goldstone modes, the well-known ferromagnons (FM). Their dispersion relation for small wave numbers is

$$
\omega_{\mathrm{FM}}(\boldsymbol{k})=D \boldsymbol{k}^{2},
$$

with $D$ the spin wave stiffness, which depends on the magnetization and vanishes as the magnetization goes to zero.

The easiest way to derive this result is to consider a nonlinear sigma-model-version of the Heisenberg action 39 Neglecting fluctuations of the amplitude of the magnetization, which can be shown to be massive, one parameterizes the order parameter

$$
\boldsymbol{M}(\boldsymbol{x})=m_{0}\left(\begin{array}{c}
\pi_{1}(\boldsymbol{x}) \\
\pi_{2}(\boldsymbol{x}) \\
\sqrt{1-\boldsymbol{\pi}^{2}(\boldsymbol{x})}
\end{array}\right)
$$

and expands the action to bilinear order in $\pi_{1,2}$. If we neglect the small relativistic term with coupling constant $d$ in Eq. (1.3), the resulting quadratic form has two identical eigenvalues

$$
\lambda=\frac{m_{0}}{2}\left(a \boldsymbol{k}^{2}+H\right)
$$

For $H=0$, one has $\lambda(\boldsymbol{k} \rightarrow 0) \rightarrow 0$, which reflects the two Goldstone modes. Physically, the homogeneous transverse magnetic susceptibility diverges. This is the static manifestation of the spontaneously broken continuous symmetry. Determining the dynamics requires an additional step. One either needs to solve an appropriate Langevin equation within a classical context, $\stackrel{36}{=}$ or treat the problem quantum mechanically ${ }^{38,41}$ Either way one obtains Eq. (3.1) with $D \propto m_{0}$.

\section{B. $O\left(g_{\text {so }}^{2}\right)$ : Helimagnons}

When the spin-orbit coupling is taken into account, we have the various phases involving helical spin textures discussed in Sec. IIA. For all of these phases the relevant symmetry is the translational one. Let $T$ be the Lie group of one-dimensional translations. Then the action is invariant under $G=T \otimes T \otimes T \equiv T^{3}$.

\section{Symmetry arguments}

Consider the aligned conical state (ACS) discussed in Sec. IIB from which the unpinned helical phase emerges as a special case at $H=0 . T^{3}$ is broken down to $T^{2}$ (translations in the two directions perpendicular to
$\hat{\boldsymbol{q}}=\hat{\boldsymbol{H}})$, so we expect one Goldstone mode. At $H=0$, one expects the soft fluctuations in the ordered phase to be phase fluctuations of the helix, and one might naively expect them to be governed by an action $S=$ $\int d \boldsymbol{x}(\boldsymbol{\nabla} \phi(\boldsymbol{x}))^{2}$, with $\phi$ the phase variable, which would lead to a soft eigenvalue proportional to $\boldsymbol{k}^{2}$. However, the ordered state is also invariant under rotations of $\boldsymbol{q}$, which can be written as a phase fluctuation with a nonvanishing gradient, so this cannot be the correct answer. The lowest-order term allowed by rotational symmetry that involves the gradients perpendicular to $\boldsymbol{q}$ is of the form $\left(\nabla_{\perp}^{2} u\right)^{2}$, with $u$ a generalized phase variable, and this leads to an eigenvalue, or inverse order parameter susceptibility, proportional to the term under the square root in Eq. (1.1) 42 The dynamics again require additional considerations, which lead to a resonance frequency that is proportional to the square root of the inverse susceptibility, unlike the ferromagnetic case, and this is expressed in Eq. (1.1). 43 These results were first obtained by means of an explicit calculation for both classical and quantum helimagnets in Ref. 25.

A magnetic field breaks the rotational invariance, so a $\boldsymbol{k}_{\perp}^{2}$-term will be present in the dispersion relation. The prefactor is expected to be analytic in $H$, and we thus expect for the dispersion relation of this "conimagnon", the Goldstone model of the ACS,

$$
\omega_{\mathrm{ACS}}(\boldsymbol{k})=\sqrt{c_{\|} k_{\|}^{2}+c_{\perp}^{\prime} \boldsymbol{k}_{\perp}^{2}+c_{\perp} \boldsymbol{k}_{\perp}^{4}}
$$

with $c_{\perp}^{\prime} \propto H^{2}$

\section{Model calculation}

For the action to $O\left(g_{\mathrm{so}}^{2}\right)$, Eqs. (2.6, 2.7) constitute an exact saddle-point solution, and we can perform an explicit calculation of the Goldstone mode. A complete parameterization of fluctuations about the saddle point can be written

$$
\begin{aligned}
\boldsymbol{M}(\boldsymbol{x})= & \left(m_{0}+\delta m_{0}(\boldsymbol{x})\right)\left(\begin{array}{c}
\pi_{1}(\boldsymbol{x}) \\
\pi_{2}(\boldsymbol{x}) \\
\sqrt{1-\boldsymbol{\pi}^{2}(\boldsymbol{x})}
\end{array}\right) \\
& +\frac{m_{1}+\delta m_{1}(\boldsymbol{x})}{\sqrt{1+\psi^{2}(\boldsymbol{x})}}\left(\begin{array}{c}
\cos \left(q z+\varphi_{0}(\boldsymbol{x})\right) \\
\sin \left(q z+\varphi_{0}(\boldsymbol{x})\right) \\
\psi(\boldsymbol{x})
\end{array}\right) .
\end{aligned}
$$

The first term is the nonlinear sigma model for the homogeneous magnetization from Sec. III A, and the second one parameterizes fluctuations of the helix in terms of an amplitude modulation, a phase $\varphi_{0}$, and a third component $\psi$. The amplitude fluctuations one expects to be massive, and an explicit calculation confirms this, so we drop $\delta m_{0}$ and $\delta m_{1}$. The field $\psi(\boldsymbol{x})$ is conveniently written

$$
\psi(\boldsymbol{x})=\varphi_{+}(\boldsymbol{x}) \cos (\boldsymbol{q} \cdot \boldsymbol{x})+\varphi_{-}(\boldsymbol{x}) \sin (\boldsymbol{q} \cdot \boldsymbol{x}),
$$


which ensure that $\varphi_{ \pm}$and $\varphi_{0}$ at zero wave number both correspond to $\boldsymbol{M}$ at wave number $q$. Double counting is avoided by restricting the theory to wave numbers small compared to $q$. If we use Eq. (3.5) in the action to $O\left(g_{\mathrm{so}}^{2}\right)$, Eqs. (1.311.4a), and expand to bilinear order in the fluctuations $\left(\phi_{1}, \phi_{2}, \phi_{3}, \phi_{4}, \phi_{5}\right) \equiv\left(\varphi_{0}, \varphi_{+}, \varphi_{-}, \pi_{1}, \pi_{2}\right)$, we obtain a Gaussian action

$$
\mathcal{A}^{(2)}=\frac{a^{2} q^{4}}{2 u V} \sum_{\boldsymbol{k}} \sum_{i=1}^{5} \phi_{i}(\boldsymbol{k}) \gamma_{i j}(\boldsymbol{k}) \phi_{j}(-\boldsymbol{k}) .
$$

A sketch of the derivation, and the explicit form of the matrix $\gamma$, are given in Appendix B. Of the five eigenvalues, one goes to zero for $\boldsymbol{k} \rightarrow 0$, in agreement with the expectation from the symmetry arguments given above. It takes the form

$$
\lambda_{1}=\alpha \hat{k}_{z}^{2}+\beta \hat{\boldsymbol{k}}_{\perp}^{2}+\delta \hat{\boldsymbol{k}}_{\perp}^{4},
$$

with $\hat{\boldsymbol{k}}=\boldsymbol{k} / q$, and coefficients

$$
\begin{aligned}
\alpha & =\hat{m}_{1}^{2}, \\
\beta & =\frac{\hat{m}_{0}^{2} \hat{m}_{1}^{2}}{\left(1+\hat{m}_{1}^{2}+\hat{m}_{0}^{2}\right)}, \\
\delta & =\frac{1}{2} \hat{m}_{1}^{2} \frac{\left(1+\hat{m}_{1}^{2}\right)^{3}-\hat{m}_{0}^{2}\left(1+\hat{m}_{1}^{4}\right)+2 \hat{m}_{0}^{4} \hat{m}_{1}^{2}}{\left(1+\hat{m}_{1}^{2}+\hat{m}_{0}^{2}\right)^{3}} .
\end{aligned}
$$

Here we have defined $\hat{m}_{0,1}^{2}=u m_{0,1}^{2} / a q^{2}$. This result is consistent with Eq. (3.4). For $H=0$, which implies $\hat{m}_{0}=0$, it reduces to the helimagnon result of Ref. 25. In addition, there are four massive eigenvalues that appear in pairs. At zero wave number, they are

$$
\begin{gathered}
\lambda_{2}=\lambda_{3} \equiv \lambda_{\varphi}=\hat{m}_{1}^{2}\left[1+\hat{m}_{0}^{2}+O\left(\hat{m}_{0}^{4}\right)\right], \\
\lambda_{3}=\lambda_{4} \equiv \lambda_{\pi}=\hat{m}_{0}^{2}\left[1+\hat{m}_{1}^{2}\right]+O\left(\hat{m}_{0}^{4}\right) .
\end{gathered}
$$

We recognize $\lambda_{\varphi}$ as representing the massive helimagnon

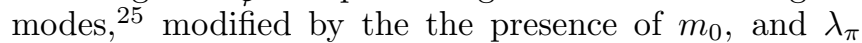
as the massive (due to the presence of a magnetic field) ferromagnons, Eq. (3.3), modified by the presence of $m_{1}$.

\section{C. $O\left(g_{\mathrm{so}}^{2}\right)$ : Skyrmionic Goldstone modes in the A-phase and the NFL region}

The helical states that have been proposed as candidates for the A-phase and were discussed in Sec. IIE are not saddle-point solutions of the action, which precludes a model calculation of the Goldstone modes resulting from this type of order. However, assuming that the order is stabilized by some mechanism, the functional form of the soft modes can still be determined by symmetry arguments analogous to those put forward in the previous subsection.

Consider a skyrmion lattice state. The state described by Eq. (2.9) is invariant only under translations in one direction, viz., the direction perpendicular to the plane of helices. The same is true for any state that is characterized by columnar order, so this property does not depend on the precise nature of the skyrmions. Any such state will thus have $\operatorname{dim}\left(T^{3} / T\right)=2$ Goldstone modes. This was to be expected: Since the skyrmions form a two-dimensional lattice, there should be two generalized phonon modes, namely, a compression mode and a shear mode. In zero magnetic field, the energy would still be invariant under global rotations of the skyrmion lattice. Hence, the soft eigenvalue can have no $k_{z}^{2}$ contribution. For $H \neq 0$ this is no longer true, and we thus expect

$$
\lambda=\alpha \boldsymbol{k}_{\perp}^{2}+\beta k_{z}^{4}+\gamma k_{z}^{2},
$$

for the soft eigenvalue, with $\gamma \propto H^{2}$, and

$$
\omega_{\mathrm{SL}}(\boldsymbol{k})=\sqrt{c_{\perp} \boldsymbol{k}_{\perp}^{2}+c_{z}^{\prime} k_{z}^{2}+c_{z} k_{z}^{4}},
$$

for the dispersion relation, with $c_{\perp}, c_{z}=O(1)$ and $c_{z}^{\prime}=$ $O\left(H^{2}\right)$.

If the NFL region can be interpreted as a molten Acrystal, see Sec. IID then one of the two Goldstone modes, the compression mode, will persist as long as their is columnar structure. This is analogous to the fact that longitudinal phonons, or ordinary sound, exist in a liquid, whereas in a crystal one has transverse phonons or shear modes in addition. The NFL region extends to $H=0$, where the dispersion relation of the compressional Goldstone mode is given by Eq. (3.11) with $c_{z}^{\prime}=0$ to first order in $g_{\mathrm{so}}$.

\section{D. $O\left(g_{\mathrm{so}}^{4}\right)$ : Effects of the crystal-field terms}

\section{Symmetry arguments}

Now consider the crystal-field terms in the action that first appear at $O\left(g_{\mathrm{so}}^{4}\right)$, Eq. (1.5a). For simplicity, let us consider the term with coupling constant $b$. It breaks rotational invariance, which invalidates the argument that leads to the absence of a $\boldsymbol{k}_{\perp}^{2}$-term in the soft mode resonance frequency. The system must remain stable regardless of the sign of $b$, and we thus expect for the dispersion relation of the helimagnons in the pinned helical state

$$
\omega_{\mathrm{HM}}(\boldsymbol{k})=\sqrt{c_{\|} k_{\|}^{2}+c_{\perp}^{\prime \prime} \boldsymbol{k}_{\perp}^{2}+c_{\perp} \boldsymbol{k}_{\perp}^{4}},
$$

with $c_{\perp}^{\prime \prime} \propto|b|$, which replaces Eq. (1.1). For the more general model given by Eqs. (1.2) - (1.5), $b_{1}, v$, and $H$ will also contribute to the elastic constant $c_{\perp}^{\prime \prime}$.

\section{Model calculation}

We now check this expectation by means of an explicit calculation. For the model with only the first of the crystal-field terms present, we have an exact saddlepoint solution, namely,

$$
\boldsymbol{M}_{\mathrm{sp}}(\boldsymbol{x})=m_{1}\left[\hat{\boldsymbol{e}}_{+} \cos (\boldsymbol{q} \cdot \boldsymbol{x})+\hat{\boldsymbol{e}}_{-} \sin (\boldsymbol{q} \cdot \boldsymbol{x})\right],
$$


with $\hat{\boldsymbol{e}}_{+}, \hat{\boldsymbol{e}}_{-}$, and $\boldsymbol{q}$ a dreibein,

$$
m_{1}=\sqrt{-r / u}
$$

where

$$
r=t-c q / 2,
$$

and

$$
\begin{aligned}
& \boldsymbol{q}=q \begin{cases}(1,1,1) / \sqrt{3} & \text { if } b<0 \\
(1,0,0) & \text { if } b>0,\end{cases} \\
& q= \begin{cases}c / 2(a+b / 3) & \text { if } b<0 \\
c / 2 a & \text { if } b>0 .\end{cases}
\end{aligned}
$$

The parameterization of fluctuations about this state is given by the second term in Eq. 3.5.

$$
\begin{aligned}
\boldsymbol{M}(\boldsymbol{x})= & \left(m_{1}+\delta m_{1}\right)\left[\hat{\boldsymbol{e}}_{+} \cos \left(\boldsymbol{q} \cdot \boldsymbol{x}+\varphi_{0}(\boldsymbol{x})\right)\right. \\
& \left.+\hat{\boldsymbol{e}}_{-} \sin \left(\boldsymbol{q} \cdot \boldsymbol{x}+\varphi_{0}(\boldsymbol{x})\right)+\hat{\boldsymbol{q}} \psi(\boldsymbol{x})\right],
\end{aligned}
$$

with $\psi(\boldsymbol{x})$ given by Eq. (3.6). We again drop the massive amplitude fluctuations and expand the action to quadratic order in the phase fluctuations. The Gaussian action is of the form

$$
\mathcal{A}^{(2)}\left[\varphi_{i}\right]=\frac{m_{1}^{2}}{2} \sum_{\boldsymbol{k}} \sum_{\alpha=0, \pm} \varphi_{\alpha}(\boldsymbol{k}) \Gamma_{\alpha \beta}(\boldsymbol{k}) \varphi_{\beta}(\boldsymbol{k}) .
$$

The explicit form of the matrix $\Gamma$ depends on the sign of $b$; it is given explicitly in Appendix [C. An inspection of the eigenvalues shows that in either case there is one eigenvalue $\lambda$ that vanishes as $\boldsymbol{k} \rightarrow 0$ and hence represents the Goldstone mode, as expected from the symmetry argument given above. To order $k_{\|}^{2}$ and $k_{\perp}^{4}$ we find

$$
\lambda=\left\{\begin{array}{rr}
a k_{\|}^{2}+\frac{1}{2} b \boldsymbol{k}_{\perp}^{2}++\frac{1}{2}(a+b) \boldsymbol{k}_{\perp}^{4} / q^{2} & \text { if } b>0, \\
(a+b / 3) k_{\|}^{2}+\frac{2}{3}|b| \boldsymbol{k}_{\perp}^{2}+\frac{1}{2}\left(a+b+O\left(g_{\mathrm{so}}^{4}\right)\right) \boldsymbol{k}_{\perp}^{4} / q^{2} & \text { if } b<0 .
\end{array}\right.
$$

In the case $b<0$ we have neglected terms proportional to $b^{2}=O\left(g_{\mathrm{so}}^{4}\right)$ in the prefactor of $\boldsymbol{k}_{\perp}^{4} / q^{2}$. This result agrees with the functional form obtained by symmetry arguments alone, Eq. (3.1), and for $b=0$ it correctly reduces to the result for the isotropic model, Ref. 25 and Eq. (1.1).

\section{Generalized helimagnons}

We can now summarize the results for the single-helix phases discussed above as follows. In the helical and conical phases, including pinning effects, there is a single Goldstone mode with a resonance frequency

$$
\omega_{0}(\boldsymbol{k})=\sqrt{c_{\|} k_{\|}^{2}+\tilde{c}_{\perp} \boldsymbol{k}_{\perp}^{2}+c_{\perp} \boldsymbol{k}_{\perp}^{4}},
$$

with $\tilde{c}_{\perp}=O\left(H^{2}, g_{\mathrm{so}}^{2}\right)$ small compared to $c_{\|}$and $c_{\perp}$. This comprises Eqs. (3.4) and (3.12).

\section{A-phase, and NFL region}

In the A-phase, pinning effects are weaker than in the helical phase due to the hexagonal nature of the skyrmion lattice, see Sec. IIE 4 above. When this weak pinning is taken in to account, the Goldstone mode is thus given by Eq. (3.11) with $c_{z}^{\prime}=O\left(g_{\mathrm{so}}^{6}, H^{2}\right)$. While in MnSi the A-phase is observed only in an external magnetic field, there is no intrinsic reason why in some other system it could not be stable in a zero field. The Goldstone modes in such a system would be given by Eq. (3.11) with an extremely small $c_{z}^{\prime}$. By the same argument, in the NFL region at $H=0$ we expect a Goldstone mode given by Eq. (3.11) with $c_{z}^{\prime}=O\left(g_{\mathrm{so}}^{6}\right)$.

\section{E. Summary of Goldstone modes, and temperature regimes}

To summarize, we have found that the (single) Goldstone mode in the pinned helical and conical phases is given by Eq. (3.17). In the pinned helical phase the parallel direction is determined by the crystal-field effects that pin the helix; in the conical phase, it is the direction of the magnetic field (which we have chosen to be the $z$-direction for all of our considerations). The elastic constant $\tilde{c}_{\perp}$ is small compared to the other elastic constants. In the pinned helical phase it is of $O\left(g_{\mathrm{so}}^{2}\right)$, and in the conical phase it is of $O\left(H^{2}\right)$. By contrast, $c_{\|}$and $c_{\perp}$ are of $O\left(g_{\mathrm{so}}\right)$ and $O\left(g_{\mathrm{so}}^{0}\right)$, and of $O\left(H^{0}\right)$, respectively. In the Aphase, there are two Goldstone modes whose dispersion relation is given by Eq. (3.11). The elastic constant $c_{z}^{\prime}$ is small of $O\left(H^{2}\right)$ and $O\left(g_{\mathrm{so}}^{6}\right)$ compared to the others. In the NFL region, the single Goldstone mode is also given by Eq. (3.11), with $c_{z}^{\prime}=O\left(g_{\mathrm{so}}^{6}\right)$. Finally, in the perpendicular conical state discussed in Appendix $\mathrm{A}$ there is a single Goldstone mode that in the absence of crystal-field effects is given by Eq. (A3). If pinning by crystal-field terms is taken into account there is an additional term $c_{y} k_{y}^{2}$ under the square root, with $c_{y}=O\left(g_{\mathrm{so}}^{2}\right)$. All of these results are also summarized in Table 1 in Sec. V.

The structures of the various Goldstone modes, and the fact that the various elastic coefficients have very different magnitudes, leads to the formation of different temperature regimes that are dominated by different physics. We now explain this using the generalized helimagnons, Eq. (3.17), as an example; the argument for the other cases works analogously. As far as the coupling of the magnetic Goldstone mode to the electronic degrees of freedom is concerned, the resonance frequency $\omega_{0}$ scales as the temperature, $\omega_{0} \sim T$. If we scale $k_{\|}$with $T / \sqrt{c_{\|}}$ and $k_{\perp}$ with $\sqrt{T} / c_{\perp}^{1 / 4}$, we obtain

$$
\omega_{0}(\boldsymbol{k})=T \sqrt{k_{\|}^{2}+\boldsymbol{k}_{\perp}^{2} \tilde{c}_{\perp} / T \sqrt{c_{\perp}}+\boldsymbol{k}_{\perp}^{4}},
$$

where $k$ now denotes the scaled, dimensionless, wave number. For $T \gg \tilde{c}_{\perp} / \sqrt{c_{\perp}}$ the symmetry-breaking $\boldsymbol{k}_{\perp}^{2}$ 
term is negligible, and the Goldstone mode is effectively what it would be in a rotationally invariant system. In this regime the physics is dominated by universal hydrodynamic effects that are independent of the microscopic details of the solid and analogous to the corresponding effects in liquid crystals. In the opposite limit, $T \ll \tilde{c}_{\perp} / \sqrt{c_{\perp}}$, the crystal-field effects due to the underlying ionic lattice, or the external magnetic field, if present, dominate and the Goldstone mode has the same functional form as (anisotropic) acoustic phonons. Due to the smallness of $\tilde{c}_{\perp}$ the universal hydrodynamic regime is sizable, and it is in this region that the most interesting effects of the magnetic order manifest themselves in the electronic properties of the system. This is true especially if the Goldstone mode appears in zero magnetic field and the pinning is very small, such as in the NFL region or a (so far hypothetical) A-phase in zero field.

\section{EFFECTS OF GOLDSTONE MODES ON ELECTRONIC PROPERTIES}

Via a coupling to the conduction electrons, the Goldstone modes derived in the preceding section influence the electronic properties of the helical magnet. In this section we derive the consequences for the specific heat, the single-particle relaxation rate, and the thermal and electrical resistivities. In all cases we consider the contribution of the Goldstone mode in isolation; it comes in addition to all other contributions to these observables.

\section{A. Specific heat}

Any well-defined (i.e., not overdamped) excitation with a dispersion relation $\omega(\boldsymbol{k})$ yields a contribution to the specific heat $C$ given by

$$
C(T)=\frac{\partial}{\partial T} \frac{1}{V} \sum_{\boldsymbol{k}} \omega(\boldsymbol{k}) n_{\mathrm{B}}(\omega(\boldsymbol{k})) .
$$

Here $n_{\mathrm{B}}(x)=1 /(\exp (x / T)-1)$ is the Bose distribution function, $V$ is the system volume, and we use units such that $\hbar=k_{\mathrm{B}}=1$. This allows one to determine the contributions to the specific heat by the various Goldstone modes.

\section{Generalized helimagnons}

We first consider the helical and aligned conical phase. The dispersion relation is given by Eq. (3.17). Performing the integral in Eq. (4.1) yields

$$
C(T)=\text { const. } \times\left\{\begin{array}{ll}
T^{3} / \sqrt{c_{z}} \tilde{c}_{\perp} & \text { if } T \ll \tilde{c}_{\perp} / \sqrt{c_{\perp}} \\
T^{2} / \sqrt{c_{z} c_{\perp}} & \text { if } T \gg \tilde{c}_{\perp} / \sqrt{c_{\perp}}
\end{array},\right.
$$

The universal hydrodynamic result, $C(T) \propto T^{2}$, was first derived in Ref. 27. It is subleading to, but distinct from, the Fermi-liquid result $C(T) \propto T+O\left(T^{3} \ln T\right)$. At asymptotically low temperatures it crosses over to a $T^{3}$ behavior consistent with the acoustic-phonon-like dispersion relation in either the pinned helical phase or the aligned conical phase at asymptotically small wave numbers.

\section{A-phase}

In the skyrmion-lattice state we find from Eq. (3.11) in conjunction with Eq. (4.1)

$$
C(T)=\text { const. } \times \begin{cases}T^{3} / c_{\perp} c_{z}^{\prime} & \text { if } T \ll c_{z}^{\prime} / \sqrt{c_{z}} \\ T^{5 / 2} / c_{\perp} c_{z}^{1 / 4} & \text { if } T \gg c_{z}^{\prime} / \sqrt{c_{z}} .\end{cases}
$$

\section{B. Relaxation times and transport coefficients}

The temperature dependence of the single-particle relaxation rate, as well as that of the electrical transport relaxation rate or the electrical resistivity, can be obtained by using the results of Refs. 28,29. The former paper obtained the following expression for the single-particle relaxation rate,

$$
\begin{aligned}
\frac{1}{\tau(\boldsymbol{k})}=2 \int_{-\infty}^{\infty} \frac{d u}{\sinh (u / T)} & \frac{1}{V} \sum_{\boldsymbol{p}} V^{\prime \prime}(\boldsymbol{p}-\boldsymbol{k} ; \boldsymbol{k}, \boldsymbol{p} ; u) \\
& \times \delta\left(u-\omega_{1}(\boldsymbol{p})\right) .
\end{aligned}
$$

Here the quasiparticle momentum $\boldsymbol{k}$ is taken to be on the 1-Fermi surface, $\omega_{1}(\boldsymbol{k})=0$, with

$$
\omega_{1,2}(\boldsymbol{k})=\frac{1}{2}\left[\xi_{\boldsymbol{k}+\boldsymbol{q}}+\xi_{\boldsymbol{k}} \pm \sqrt{\left(\xi_{\boldsymbol{k}+\boldsymbol{q}}-\xi_{\boldsymbol{k}}\right)^{2}+4 \lambda^{2}}\right],
$$

which is separated from the 2-Fermi surface, $\omega_{2}(\boldsymbol{k})=0$, by twice the Stoner gap $\lambda$. Here

$$
\xi_{\boldsymbol{k}}=\epsilon_{\boldsymbol{k}}-\epsilon_{\mathrm{F}}
$$

with

$$
\epsilon_{\boldsymbol{k}}=\boldsymbol{k}^{2} / 2 m_{\mathrm{e}}+\frac{\nu}{2 m_{\mathrm{e}} k_{\mathrm{F}}^{2}}\left(k_{x}^{2} k_{y}^{2}+k_{y}^{2} k_{z}^{2}+k_{z}^{2} k_{x}^{2}\right)
$$

an electronic energy-momentum relation consistent with a cubic crystal. The dimensionless parameter $\nu$ is a measure of deviations from a nearly-free electron model. $V^{\prime \prime}$ is the spectrum of an effective potential given by

$$
V\left(k ; \boldsymbol{p}_{1}, \boldsymbol{p}_{2}\right)=\frac{1}{2} \lambda^{2} \chi(k) \tilde{\gamma}\left(\boldsymbol{k}, \boldsymbol{p}_{1}\right) \tilde{\gamma}\left(-\boldsymbol{k}, \boldsymbol{p}_{2}\right),
$$

with vertices

$$
\begin{gathered}
\tilde{\gamma}(\boldsymbol{k}, \boldsymbol{p})=\frac{q}{8 m_{\mathrm{e}} \lambda}\left[k_{z}+\frac{\nu}{k_{\mathrm{F}}^{2}}\left(k_{z} \boldsymbol{p}_{\perp}^{2}+2\left(\boldsymbol{k}_{\perp} \cdot \boldsymbol{p}_{\perp}\right) p_{z}\right)\right] \\
+O\left(k^{2}\right),
\end{gathered}
$$


and $\chi$ the soft-mode susceptibility,

$$
\chi(k)=\frac{1}{2 N_{\mathrm{F}}} \frac{q^{2}}{3 k_{\mathrm{F}}^{2}} \frac{1}{\omega_{0}^{2}(\boldsymbol{k})-(i \Omega)^{2}} .
$$

Here $\omega_{0}(\boldsymbol{k})$ is the resonance frequency, which is equal to the frequency given in Eq. (3.4), (3.11), (3.12), or (A3), depending on the phase under consideration. The electrical transport relaxation time, which determines the electrical resistivity, is effectively given by averaging a similar expression over the Fermi surface, $\stackrel{29}{\underline{9}}$

$$
\begin{aligned}
\frac{1}{\tau_{\mathrm{el}}^{\mathrm{tr}}}= & \frac{1}{N_{\mathrm{F}}} \int_{-\infty}^{\infty} \frac{d u}{\sinh (u / T)} \frac{1}{V^{2}} \sum_{\boldsymbol{p}, \boldsymbol{k}} \frac{(\boldsymbol{p}-\boldsymbol{k})^{2}}{k_{\mathrm{F}}^{2}} \\
& \times V^{\prime \prime}(\boldsymbol{p}-\boldsymbol{k} ; \boldsymbol{k}, \boldsymbol{p} ; u) \delta\left(u-\omega_{1}(\boldsymbol{p})\right) \delta\left(\omega_{1}(\boldsymbol{k})\right) .
\end{aligned}
$$

Note the additional, compared to Eq. (4.4), factor of $(\boldsymbol{p}-\boldsymbol{k})^{2}$ under the integral in Eq. (4.7). This is characteristic of the description of electrical transport in a Boltzmann approximation and leads to a temperature dependence of the electrical resistivity that is different from that of the single-particle relaxation rate $\underline{\underline{44}, 45} \mathrm{In}$ contrast, in a Boltzmann description of thermal transport this additional factor is absent, and the temperature dependence of the thermal conductivity is given by that of the single-particle relaxation rate $\underline{\underline{45}}$

We note that these expressions for $1 / \tau(\boldsymbol{k})$ and $1 / \tau_{\mathrm{el}}^{\mathrm{tr}}$ vanish as $q \rightarrow 0$. While they give the leading asymptotic temperature dependence for the relaxation rates in helimagnets, they therefore cannot be used to obtain the corresponding results in the ferromagnetic limit. The asymptotic low- $T$ behavior of the rates in ferromagnets is qualitatively different and briefly treated in Appendix D In the context of helimagnets, for a non-spherical Fermi surface (i.e., $\nu \neq 0$ in Eq. (4.5c)), and for generic wave vectors $\boldsymbol{k}$, the temperature scaling behavior of the single-particle relaxation rate $1 / \tau \equiv 1 / \tau(\boldsymbol{k})$, the thermal resistivity $\rho_{\text {th }}$, and the electrical resistivity $\rho_{\text {el }}$ can be represented schematically by the expressions

$$
\begin{aligned}
\frac{1}{\tau} \sim \rho_{\mathrm{th}} \sim \int & d p_{\|} \int d \boldsymbol{p}_{\perp}^{2} \frac{\boldsymbol{p}_{\perp}^{2}+p_{\|}^{2}}{\sinh \left(\omega_{0}(\boldsymbol{p}) / T\right)} \\
& \times \frac{\delta\left(\omega_{0}(\boldsymbol{p})-p_{\perp}-p_{\|}\right)}{\omega_{0}(\boldsymbol{p})} . \\
\rho_{\mathrm{el}} \sim \int d p_{\|} \int & d \boldsymbol{p}_{\perp}^{2} \frac{\left(\boldsymbol{p}_{\perp}^{2}+p_{\|}^{2}\right)^{2}}{\sinh \left(\omega_{0}(\boldsymbol{p}) / T\right)} \\
& \times \frac{\delta\left(\omega_{0}(\boldsymbol{p})-p_{\perp}-p_{\|}\right)}{\omega_{0}(\boldsymbol{p})} .
\end{aligned}
$$

The resonance frequency always scales as the temperature, $\omega_{0} \sim T$, and the temperature dependence of the relaxation rates thus is determined by how the momentum components scale with temperature.

\section{Generalized helimagnons}

For the helical and conical phases we have, from Eq. (3.17), $p_{\|} \sim T$, and $p_{\perp} \sim T$ and $\sim T^{1 / 2}$ at asymptotically low and intermediate temperatures, respectively. This yields the following temperature dependence for the single-particle relaxation rate and the thermal resistivity:

$$
\frac{1}{\tau} \propto \rho_{\text {th }} \propto\left\{\begin{array}{ll}
T^{3} & \text { if } T \ll \tilde{c}_{\perp} / \sqrt{c_{\perp}} \\
T^{3 / 2} & \text { if } T \gg \tilde{c}_{\perp} / \sqrt{c_{\perp}}
\end{array} .\right.
$$

The corresponding result for the electrical resistivity is

$$
\rho_{\mathrm{el}} \propto\left\{\begin{array}{ll}
T^{5} & \text { if } T \ll \tilde{c}_{\perp} / \sqrt{c_{\perp}} \\
T^{5 / 2} & \text { if } T \gg \tilde{c}_{\perp} / \sqrt{c_{\perp}}
\end{array} .\right.
$$

In a vanishing external field, and in a temperature regime where pinning effects are not relevant, we recover the $T^{3 / 2}$ and $T^{5 / 2}$ behavior for $1 / \tau$ and $\rho_{\mathrm{el}}$, respectively, of Ref. 27 and 29 .

\section{A-phase}

For a skyrmion lattice, we obtain by using Eq. (3.11)

$$
\frac{1}{\tau} \propto \rho_{\mathrm{th}} \propto\left\{\begin{array}{ll}
T^{3} & \text { if } T \ll c_{z}^{\prime} / \sqrt{c_{z}} \\
T^{2} & \text { if } T \gg c_{z}^{\prime} / \sqrt{c_{z}}
\end{array} .\right.
$$

for the single-particle rate, and

$$
\rho_{\mathrm{el}} \propto\left\{\begin{array}{ll}
T^{5} & \text { if } T \ll c_{z}^{\prime} / \sqrt{c_{z}} \\
T^{3} & \text { if } T \gg c_{z}^{\prime} / \sqrt{c_{z}}
\end{array} .\right.
$$

for the electrical resistivity.

\section{Systems with quenched disorder}

The preceding results hold for clean systems. In the presence of quenched disorder, elastic scattering of the conduction electrons leads to profound effects that manifest themselves in the transport properties. One needs to distinguish between the strong-disorder regime, where the transport is diffusive, and the weak-disorder regime, where it is ballistic. In a Fermi liquid, these two regimes are characterized by $T \tau \ll 1$ and $T \tau \gg 1$, respectively $\underline{\underline{46}}$ In a helical magnet, the weak-disorder or ballistic regime is characterized by $\stackrel{28,29}{2}$

$$
\sqrt{\left(\epsilon_{\mathrm{F}} \tau_{\mathrm{el}}\right)^{2} T / \lambda} \gg 1,
$$

where $\tau_{\mathrm{el}}$ is the elastic relaxation time. In this regime there is an additional contribution $\delta(1 / \tau)$ to the relaxation rates that is qualitatively the same for both the single-particle rate and the electron transport rate, $\stackrel{47}{=}$ and thus provides the temperature dependence of the corrections to both the electrical and thermal resistivities. It 
was shown in Refs. 28, 29 that, for temperature scaling purposes, this contribution can be represented by

$$
\begin{aligned}
& \delta(1 / \tau) \propto \delta \rho_{\mathrm{el}} \propto \delta \rho_{\mathrm{th}} \\
& \quad \sim \int d u n_{\mathrm{F}}(u / T) \int d p_{\|} \int d p_{\perp}^{2} \frac{1}{\omega_{0}(\boldsymbol{p})} \delta\left(u-\omega_{0}(\boldsymbol{p})\right) .
\end{aligned}
$$

\section{Generalized helimagnons}

From Eq. (4.14) we see that at temperatures where pinning effects are not important, generalized helimagnons lead to $\delta(1 / \tau) \propto T$, a result first obtained in Ref. 29. At asymptotically low temperatures, characterized by $T \ll \tilde{c}_{\perp} / \sqrt{c_{\perp}}$, one finds a $T^{2}$-behavior. In the pinned helical phase the crossover temperature between these two regimes is determined by the strength of the crystalfield effects; in the conical phase the magnetic field also cuts off the universal hydrodynamics $T$-behavior.

\section{A-phase}

For the A-phase, Eq. (3.11) yields

$$
\delta(1 / \tau) \propto\left\{\begin{array}{ll}
T^{2} & \text { if } T \ll c_{z}^{\prime} / \sqrt{c_{z}} \\
T^{3 / 2} & \text { if } T \gg c_{z}^{\prime} / \sqrt{c_{z}}
\end{array} .\right.
$$

The pinning effects in the A-phase are very weak due to the hexagonal nature of the skyrmion lattice, with $c_{z}^{\prime}$ only of $O\left(g_{\mathrm{so}}^{6}\right)$, see Secs. IIE4 4and IID 4 The size of the asymptotic region is therefore likely to be dominated by the $H$-dependence of $c_{z}^{\prime}$. Whether or not the universal hydrodynamic $T^{3 / 2}$-behavior is observable in the $\mathrm{A}$-phase (there currently are no experimental indications that it is) would require a detailed quantitative analysis that goes beyond the scope of the current paper.

\section{NFL region}

The preceding result is also of interest with respect to the non-Fermi-liquid region shown in Fig. 2, which is not a phase with long-range order, but where the electrical conductivity shows a pronounced $T^{3 / 2}$-behavior $\stackrel{15}{\stackrel{15}{ } \mathrm{An}}$ explanation that has recently been proposed ${ }^{16}$ is as follows. The $T^{3 / 2}$-behavior derived above is a consequence of the structure of the Goldstone modes due to columnar fluctuations, Eq. (3.11), in conjunction with weak quenched disorder. In the A-phase, which displays longrange order in the form of a skyrmion lattice, there are two such Goldstone modes, see Sec. IIIC, If the NFL region can be interpreted as a melted skyrmion lattice, then the resulting skyrmion fluid will still have one Goldstone mode with the same structure, namely, the compression mode mentioned in Sec. IIIC. Weak quenched disorder will then still produce a contribution to the electrical resistivity, as well as to the single-particle relaxation rate, that is proportional to $T^{3 / 2}$ in a pre-asymptotic region. The NFL region is observed to extend to a vanishing external magnetic field, so the low- $T$ boundary of the universal hydrodynamic region is determined by the pinning effects, which are very weak, see Secs. IIE4 and IIID4 The universal hydrodynamic $T^{3 / 2}$ behavior is therefore expected to extend to very low temperatures. A remaining question is the size of the prefactor, which in a bare theory is expected to be small due to the long length scale set by the skyrmion lattice. The resolution proposed in Ref. 16 is that mode-mode coupling effects drastically enhance the magnitude of the effect, in analogy to what is believed to happen in the blue phases of liquid crystals $\underline{\underline{48}-\underline{50}}$

\section{SUMMARY, AND CONCLUSION}

In summary, we have given a comprehensive description of all phases in Dzyaloshinsky-Moriya helical magnets where long-range order has been observed. These include, the pinned helical phase at weak magnetic fields, the conical phase at higher magnetic fields, and the Aphase at intermediate magnetic fields and temperatures close to the critical temperature. We have shown that the system goes from the conical phase at high magnetic field to the pinned helical phase at low magnetic field via two distinct phase transitions; a second-order transition where the orientation of the helix changes smoothly, followed by a first-order transition where both the orientation and the polarization of the helix change discontinuously. For the A-phase we have considered the recent interpretation, based on the observation of a six-fold symmetry in the neutron scattering signature, as a hexagonal lattice of skyrmionic line defects. We have also discussed the perpendicular conical state, which had been discussed earlier as a possible realization of the A-phase. In addition, we have discussed a proposal for the interpretation of the non-Fermi-liquid (NFL) region in the disordered phase, which does not display long-range magnetic order but has many features in common with the A-phase. For all of these states we have determined the number and nature of the Goldstone modes, and the temperature dependencies of various observables that result from the scattering of the conduction electrons by these excitations. If the state in question can be described by an exact saddlepoint solution of a model Hamiltonian we have provided an explicit calculation; in other cases we have used symmetry arguments to determine the functional form of the Goldstone modes. The results are summarized in Table I. The Goldstone modes show unusual anisotropic frequency-momentum relations that result from helical spin structures in the various states and are reminiscent of the Goldstone modes in smectic and cholesteric liquid crystals. The specific heat $C(T)$, the single-particle relaxation rate $1 / \tau(T)$ and the thermal resistivity $\rho_{\mathrm{th}}(T)$, 


\begin{tabular}{|c|c|c|c|c|c|c|}
\hline & & & $\begin{array}{c}\text { pinned helical } \\
\text { /conical }\end{array}$ & $\begin{array}{c}\text { A-phase } \\
(\boldsymbol{H} \| \hat{\boldsymbol{z}})\end{array}$ & $\begin{array}{c}\mathrm{PCS}^{a} \\
(\boldsymbol{H}\|\hat{\boldsymbol{z}}, \boldsymbol{q}\| \hat{\boldsymbol{x}})\end{array}$ & $\mathrm{NFL}^{b}$ \\
\hline & \multirow{2}{*}{$\begin{array}{c}\text { Goldstone } \\
\text { modes }\end{array}$} & $\omega_{0}(\boldsymbol{k})$ & $\sqrt{c_{\| \mid} k_{\|}^{2}+\tilde{c}_{\perp} \boldsymbol{k}_{\perp}^{2}+c_{\perp} \boldsymbol{k}_{\perp}^{4}}$ & $\sqrt{c_{z}^{\prime} k_{z}^{2}+c_{z} k_{z}^{4}+c_{\perp} \boldsymbol{k}_{\perp}^{2}}$ & $\sqrt{c_{x} k_{x}^{2}+c_{z} k_{z}^{2}+c_{\perp} \boldsymbol{k}_{\perp}^{4}}$ & $\sqrt{c_{||} k_{\|}^{4}+c_{\perp} \boldsymbol{k}_{\perp}^{2}}$ \\
\hline & & \# & 1 & 2 & 1 & 1 \\
\hline \multirow{6}{*}{$\frac{\widetilde{\varpi}}{0}$} & \multirow{2}{*}{$C(T)$} & $\begin{array}{l}\text { universal hydro- } \\
\text { dynamic regime }\end{array}$ & $T^{2}$ & $T^{5 / 2}$ & $T^{2}$ & \multirow{2}{*}{$T^{5 / 2}$} \\
\hline & & $\begin{array}{l}\text { crystal-field } \\
\text { regime }(T \rightarrow 0)\end{array}$ & $T^{3}$ & $T^{3}$ & $T^{5 / 2}$ & \\
\hline & $1 / \tau(T)$ & $\begin{array}{l}\text { universal hydro- } \\
\text { dynamic regime }\end{array}$ & $T^{3 / 2}$ & $T^{2}$ & $T^{3 / 2}$ & \multirow{2}{*}{$T^{2}$} \\
\hline & $\rho_{\mathrm{th}}(T)$ & $\begin{array}{l}\text { crystal-field } \\
\text { regime }(T \rightarrow 0)\end{array}$ & $T^{3}$ & $T^{3}$ & $T^{2}$ & \\
\hline & \multirow{2}{*}{$\rho_{\mathrm{el}}(T)$} & $\begin{array}{l}\text { universal hydro- } \\
\text { dynamic regime }\end{array}$ & $T^{5 / 2}$ & $T^{3}$ & $T^{5 / 2}$ & \multirow{2}{*}{$T^{3}$} \\
\hline & & $\begin{array}{l}\text { crystal-field } \\
\text { regime }(T \rightarrow 0)\end{array}$ & $T^{5}$ & $T^{5}$ & $T^{3}$ & \\
\hline \multirow[b]{2}{*}{ 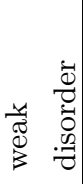 } & $\delta \rho_{\mathrm{el}}(T)$ & $\begin{array}{l}\text { universal hydro- } \\
\text { dynamic regime }\end{array}$ & $T$ & $T^{3 / 2}$ & $T$ & \multirow{2}{*}{$T^{3 / 2}$} \\
\hline & $\delta \rho_{\mathrm{th}}(T)$ & $\begin{array}{l}\text { crystal-field } \\
\text { regime }^{c}\end{array}$ & $T^{2}$ & $T^{2}$ & $T^{2}$ & \\
\hline
\end{tabular}

${ }^{a}$ This phase, the perpendicular conical state, has not been experimentally observed so far.

${ }^{b}$ The crystal-field effects are much smaller in the NFL region than in the ordered phases, see Secs. IIE 4 and IID4 We results listed are valid in the universal hydrodynamic regime, which is expected to extend to very low temperatures; the true asymptotic low- $T$ behavior is the same as in the A-phase, where the size of the $T \rightarrow 0$ region is determined by the external magnetic field.

${ }^{c}$ In the presence of quenched disorder this regime does not represent the true asymptotic $T \rightarrow 0$ behavior, which is characterized by diffusive rather than ballistic electron dynamics, see the table caption and Eq. 4.13).

TABLE I: Properties of various ordered phases and the proposed state representing the non-Fermi-liquid region. Listed are the number of Goldstone modes and their respective dispersion relations, as well as the temperature dependence of various observables. The elastic constants $\tilde{c}_{\perp}, c_{z}^{\prime}$, and $c_{z}$ in the Goldstone modes for the pinned helical/conical phase, the A-phase, and the PCS, respectively, are due to crystal-field effects or an external magnetic field and are small compared to the other elastic constants. The universal hydrodynamic regime is the temperature region where crystal-field effects are not important and these elastic constants can be neglected. The true asymptotic behavior as $T \rightarrow 0$ is dominated by the crystal-field effects and is realized only at very low temperatures. In the presence of weak disorder, the regime dominated by crystal-field effects is bounded below as well as above and does not represent the true asymptotic low-temperature regime. See the text for more information.

and the electrical resistivity $\rho_{\mathrm{el}}(T)$, all display temperature dependencies that are distinct from, and in some cases stronger than, those in a Fermi liquid. The most remarkable result is a non-Fermi liquid $T^{3 / 2}$ behavior of the electrical resistivity in certain temperature regimes in the A-phase and in the NFL region, which is proposed as an explanation of the observed enigmatic properties of the DM magnet MnSi.

\section{Acknowledgments}

This work was initiated at the Aspen Center for Physics, and supported by the National Science Foundation under Grant Nos. DMR-09-29966 and DMR-0901907. 


\section{Appendix A: The perpendicular conical state}

Guided by earlier experimental results that did not reveal the six-fold symmetry discovered by Mühlbauer et al., ${ }^{11}$ Grigoriev et al. ${ }^{10}$ had proposed a perpendicular conical state (PCS) to represent the A-phase, i.e., a spin configuration of the form

$$
\boldsymbol{M}(\boldsymbol{x})=m_{0} \hat{\boldsymbol{H}}+m_{1}\left[\hat{\boldsymbol{e}}_{+} \cos (\boldsymbol{q} \cdot \boldsymbol{x})+\hat{\boldsymbol{e}}_{-} \sin (\boldsymbol{q} \cdot \boldsymbol{x})\right]
$$

with $\boldsymbol{q}$ perpendicular to $\boldsymbol{H}$ and $\hat{\boldsymbol{e}}_{+}, \hat{\boldsymbol{e}}_{-}$, and $\hat{\boldsymbol{q}}$ forming a dreibein. This state is not a saddle point either, and Ref. 11 found it energetically unfavorable compared to the conical and skyrmion lattice states. However, it can be stabilized, at least in principle, and we therefore include it in our discussion. Ref. 10 proposed that the PCS is stabilized due to a gap in the helimagnon excitation spectrum, which in turn had been proposed theoretically in Ref. 51. Such a gap is at odds with Goldstone's theorem as well as with the calculation in Ref. 25. A different possibility, which is consistent with symmetry considerations, is a large negative value of the parameter $w$ in Eq. (1.3), which stabilizes the PCS in a parameter regime close to where the A-phase is observed, see Fig. 8. While such a large value of $w$ is not realistic, this illustrates that the PCS can be stabilized by terms in the action that are allowed by symmetry, and it therefore is useful to determine the properties of this state.

Let us now consider the Goldstone modes in the PCS, using the symmetry arguments explained in Sec. III A. For definiteness, we assume that the $\boldsymbol{q}$-vector points in the $x$-direction. The ordered state is still invariant under translations in the $y$ and $z$-directions, so we have $\operatorname{dim}\left(T^{3} / T^{2}\right)=1$ Goldstone mode. With the magnetic field $H$ in the $z$-direction, the state is still invariant under rotations of $\boldsymbol{q}$ in the $x$ - $y$-plane. The eigenvalue therefore cannot have a $k_{y}^{2}$ term. The soft eigenvalue therefore must have the form

$$
\lambda=\alpha k_{x}^{2}+\beta\left(k_{y}^{4}+k_{z}^{4}\right)+\gamma k_{z}^{2},
$$

where $\gamma \propto H^{2}$ for small $H$. The structure of the dynamics will be the same as in the aligned conical case, and we thus have a dispersion relation

$$
\omega_{\mathrm{PCS}}(\boldsymbol{k})=\sqrt{c_{x} k_{x}^{2}+c_{z} k_{z}^{2}+c_{\perp}\left(k_{y}^{4}+k_{z}^{4}\right)}
$$

with $c_{z} \propto H^{2}$ and $c_{x}$ and $c_{\perp}$ constant for $H \rightarrow 0$. Here we neglect pinning effects, which lead to a $k_{y}^{2}$-term under the square root at asymptotically low wave numbers.

For the specific heat contribution we find, from Eqs. (4.1) and (A3),

$$
C(T)=\text { const. } \times \begin{cases}T^{5 / 2} /\left(c_{x} c_{z}\right)^{1 / 2} c_{\perp}^{1 / 4} & \text { if } T \ll c_{z} / \sqrt{c_{\perp}} \\ T^{2} / \sqrt{c_{x} c_{\perp}} & \text { if } T \gg c_{z} / \sqrt{c_{\perp}} .\end{cases}
$$

This result is different from the one for the skyrmionic Goldstone mode in Sec. IIIC. Measurements of the specific heat therefore offer a way to distinguish between

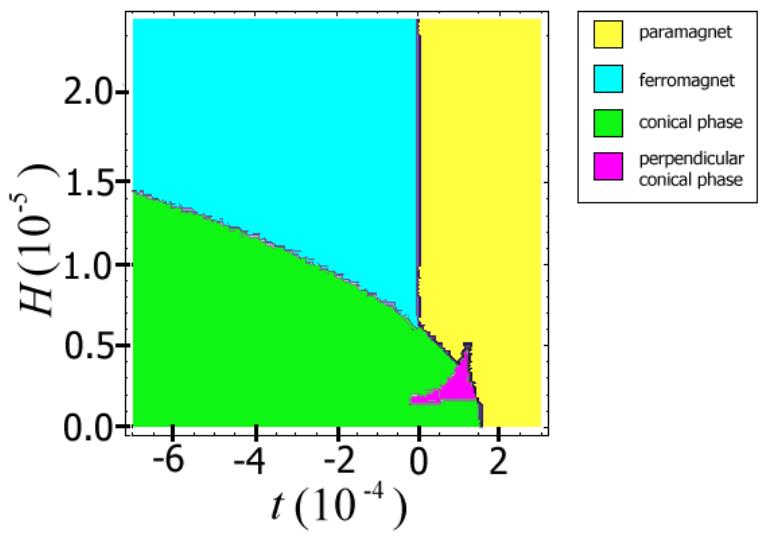

FIG. 8: Stabilization of the PCS by a large negative value of $w$. Shown are the regions of stability for various phases for the action shown in Eq. (1.3) with $a=1, d=0.025, u=0.1$, and $w=-300$.

these two states short of a direct determination of the spin structure.

Using Eq. (A3) in an obvious generalization of Eqs. (4.8) we find for the single-particle relaxation rate and the thermal resistivity

$$
\frac{1}{\tau} \propto \rho_{\mathrm{th}} \propto\left\{\begin{array}{ll}
T^{2} & \text { if } T \ll c_{z} / \sqrt{c_{\perp}} \\
T^{3 / 2} & \text { if } T \gg c_{z} / \sqrt{c_{\perp}}
\end{array},\right.
$$

and for the electrical resistivity

$$
\rho_{\mathrm{el}} \propto \begin{cases}T^{3} & \text { if } T \ll c_{z} / \sqrt{c_{\perp}} \\ T^{5 / 2} & \text { if } T \gg c_{z} / \sqrt{c_{\perp}}\end{cases}
$$

\section{Appendix B: Gaussian fluctuations in the aligned conical phase}

Here we derive the Gaussian fluctuation action in the conical phase, Eq. (3.7). The equation of state for this phase, Eqs. 2.6. 2.7), can be written

$$
t-a q^{2}+u\left(m_{0}^{2}+m_{1}^{2}\right)=0
$$

with $q$ and $m_{0}$ given by Eqs. (2.6b) and (2.6c), respectively. Inserting Eq. (3.10) into the action, Eqs. (1.3. 1.4a), dropping the amplitude fluctuations $\delta m_{0}$ and $\delta m_{1}$, and expanding to bilinear order in the phases yields Eq. (3.7) with 


$$
\gamma(\boldsymbol{k})=\left(\begin{array}{ccccc}
\hat{m}_{1}^{2} \hat{k}^{2} & -i \hat{m}_{1}^{2} \hat{k}_{y} & i \hat{m}_{1}^{2} \hat{k}_{x} & 0 & 0 \\
i \hat{m}_{1}^{2} \hat{k}_{y} & \hat{m}_{1}^{2}\left(1+\hat{m}_{0}^{2}+\frac{1}{2} \hat{k}^{2}\right) & -i \hat{m}_{1}^{2} \hat{k}_{z} & 0 & \hat{m}_{0}^{2} \hat{m}_{1}^{2} \\
-i \hat{m}_{1}^{2} \hat{k}_{x} & i \hat{m}_{1}^{2} \hat{k}_{z} & \hat{m}_{1}^{2}\left(1+\hat{m}_{0}^{2}+\frac{1}{2} \hat{k}^{2}\right) & \hat{m}_{0}^{2} \hat{m}_{1}^{2} & 0 \\
0 & 0 & \hat{m}_{0}^{2} \hat{m}_{1}^{2} & \hat{m}_{0}^{2}\left(1+\hat{m}_{1}^{2}+\hat{k}^{2}\right) & 2 i \hat{m}_{0}^{2} \hat{k}_{z} \\
0 & \hat{m}_{0}^{2} \hat{m}_{1}^{2} & 0 & -2 i \hat{m}_{0}^{2} \hat{k}_{z} & \hat{m}_{0}^{2}\left(1+\hat{m}_{1}^{2}+\hat{k}^{2}\right)
\end{array}\right) .
$$

A determination of the eigenvalues yields Eqs. (3.8, 3.9).

\section{Appendix C: Gaussian fluctuations in the pinned helical phase}

Here we derive the Gaussian fluctuation action in the pinned helical phase, Eq. (3.15). Dropping the amplitude fluctuations, and expanding to linear order in the phase fluctuations, Eq. (3.14) becomes

$$
\boldsymbol{M}(\boldsymbol{x})=\boldsymbol{M}_{\mathrm{sp}}(\boldsymbol{x})+\delta \boldsymbol{M}(\boldsymbol{x}),
$$

with

$$
\delta \boldsymbol{M}(\boldsymbol{x})=m_{1}\left[\left(-\hat{\boldsymbol{e}}_{-} \sin (\boldsymbol{q} \cdot \boldsymbol{x})+\hat{\boldsymbol{e}}_{+} \cos (\boldsymbol{q} \cdot \boldsymbol{x})\right) \varphi_{0}(\boldsymbol{x})+\hat{\boldsymbol{q}} \sin (\boldsymbol{q} \cdot \boldsymbol{x}) \varphi_{-}(\boldsymbol{x})+\hat{\boldsymbol{q}} \cos (\boldsymbol{q} \cdot \boldsymbol{x}) \varphi_{+}(\boldsymbol{x})+O\left(\varphi_{0}^{2}\right)\right] .
$$

$\boldsymbol{M}_{\mathrm{sp}}$ is an exact saddle point of the action, Eqs. (1.2) - (1.5) with $b_{1}=v=0$, so the terms linear in $\delta \boldsymbol{M}$ vanish. We now consider terms bilinear in $\varphi_{0,+,-}$ and first concentrate on the gradient-free terms. Neglecting rapidly fluctuating Fourier components proportional to $e^{i n \boldsymbol{q} \cdot \boldsymbol{x}}$ with $n \geq 2$ one finds

$$
\begin{aligned}
\mathcal{A}^{(2)}[\delta \boldsymbol{M}] / m_{1}^{2}= & \frac{1}{2} \int d \boldsymbol{x} \varphi_{0}(\boldsymbol{x})\left[t+a q^{2}-c q+u m_{1}^{2}+\frac{1}{2} b \sum_{i=1}^{3} q_{i}^{2}\left[\left(\hat{e}_{+}^{i}\right)^{2}+\left(\hat{e}_{-}^{i}\right)^{2}\right]\right] \varphi_{0}(\boldsymbol{x}) \\
& +\frac{1}{4} \int d \boldsymbol{x} \sum_{\alpha= \pm} \varphi_{\alpha}(\boldsymbol{x})\left[t+a q^{2}+u m_{1}^{2}+b q^{2} \sum_{i=1}^{3} \hat{q}_{i}^{4}\right] \varphi_{\alpha}(\boldsymbol{x}) \\
& -\frac{1}{2} b q^{2} \int d \boldsymbol{x} \sum_{\alpha= \pm} \sum_{i=1}^{3} \hat{e}_{\alpha}^{i} \hat{q}_{i}^{3} \varphi_{0}(\boldsymbol{x}) \varphi_{\alpha}(\boldsymbol{x})+\text { (gradient terms) }
\end{aligned}
$$

An explicit calculation shows that

$$
\begin{gathered}
\sum_{i=1}^{3} \hat{q}_{i}^{2}\left[\left(\hat{e}_{+}^{i}\right)^{2}+\left(\hat{e}_{-}^{i}\right)^{2}\right]=1-f(\hat{\boldsymbol{q}}), \\
\sum_{i=1}^{3} \hat{q}_{i}^{4}=f(\hat{\boldsymbol{q}})
\end{gathered}
$$

with

$$
f(\hat{\boldsymbol{q}})=\beta_{1}^{4}+\beta_{2}^{4}+\beta_{3}^{4},
$$

where $\beta_{1,2,3}$ are the direction cosines of $\hat{\boldsymbol{q}}$. Furthermore,

$$
\sum_{i=1}^{3} \hat{e}_{\alpha}^{i}\left(\hat{q}_{i}\right)^{3}=0
$$

for $\alpha=+,-$ and for both $\hat{\boldsymbol{q}}=(1,1,1) / \sqrt{3}$ and $\hat{\boldsymbol{q}}=$ $(1,0,0)$. Using the equation of state, Eqs. (3.13b, 3.13c), we see that the $\varphi_{0}-\varphi_{0}$ vertex and the $\varphi_{0}-\varphi_{\alpha}$ vertices all vanish. At zero wave number we thus have one zero eigenvalue that corresponds to one Goldstone mode, in agreement with the expectation from Sec. IIIB 1.

We next calculate the gradient-squared terms. Geometric identities similar to those expressed in Eqs. (C3) allow to determine the vertices. One finds a Gaussian action of the form given by Eq. (3.15). For $b>0$, the matrix $\Gamma$ is given by

$$
\Gamma(\boldsymbol{k})=\left(\begin{array}{ccc}
a \boldsymbol{k}^{2}+\frac{1}{2} b \boldsymbol{k}_{\perp}^{2} & -i \frac{1}{2} c k_{y} & -i \frac{1}{2} c k_{x} \\
i \frac{1}{2} c k_{y} & \frac{1}{2} c q+\frac{1}{2} a \boldsymbol{k}^{2}+\frac{1}{2} b k_{z}^{2} & i(a+b) q k_{z} \\
i \frac{1}{2} c k_{x} & -i(a+b) q k_{z} & \frac{1}{2} c q+\frac{1}{2} a \boldsymbol{k}^{2}+\frac{1}{2} b k_{z}^{2}
\end{array}\right) \quad(b>0)
$$


For $b<0$, the matrix $\Gamma$ takes the form

$$
\begin{aligned}
\Gamma_{00}(\boldsymbol{k}) & =(a+b / 3) k^{2}, \\
\Gamma_{0+}(\boldsymbol{k}) & =\Gamma_{+0}(\boldsymbol{k})^{*}=i\left(\frac{1}{2} c+\frac{1}{3} b q\right) \frac{1}{\sqrt{2}}\left(k_{\|}-3 k_{z}\right)+\frac{1}{2 \sqrt{6}} b\left(k_{x}^{2}-k_{y}^{2}\right), \\
\Gamma_{0-}(\boldsymbol{k}) & =\Gamma_{-0}(\boldsymbol{k})^{*}=i\left(\frac{1}{2} c+\frac{1}{3} b q\right) \frac{1}{\sqrt{2}}\left(k_{x}-k_{y}\right)-\frac{1}{2 \sqrt{6}} b\left(k_{x}^{2}+k_{y}^{2}-2 k_{z}^{2}\right), \\
\Gamma_{++}(\boldsymbol{k}) & =\Gamma_{--}(\boldsymbol{k})=\frac{1}{2} c q+\frac{1}{2}(a+b / 3) k^{2}, \quad(b<0) . \\
\Gamma_{+-}(\boldsymbol{k}) & =\Gamma_{-+}(\boldsymbol{k})^{*}=i(a+b / 3) q k_{\|}
\end{aligned}
$$

Calculating the eigenvalues one finds Eq. (3.16).

\section{Appendix D: Relaxation rates in ferromagnets}

In this appendix we sketch how the expressions for the single-particle and transport relaxation rates given in Sec. IVB change in the ferromagnetic limit. A more thorough discussion of this topic will be given elsewhere ${ }^{52}$

There are three major changes compared to Eqs. (4.4) and (4.7) that take place in the ferromagnetic limit. Firstly, there is no intra-Stoner-band scattering. This is because in a ferromagnet, the Goldstone mode is entirely transverse with respect to the direction of the magnetization, whereas in a helimagnet this is not the case $\underline{\underline{53}}$ As a result, with $\boldsymbol{k}$ in Eq. (4.4) on the 1-Fermi surface, the resonance frequency inside the delta function under the integral will be $\omega_{2}(\boldsymbol{p})$. Secondly, the dimensionless vertex $\tilde{\gamma}$, Eq. (4.6b), changes to unity. Technically, this is seen most easily within the formalism of Ref. 28. Physically, it reflects the fact that for intra-Stonerband scattering the magnetic fluctuations couple to the quasi-particle density, and thus physically act akin to a chemical potential, only gradients of which contribute to scattering. For inter-Stoner-band scattering, on the other hand, they physically act akin to an external magnetic field, and thus they couple without gradients. Thirdly, the form of the susceptibility $\chi$, Eq. (4.6c), changes. It now reflects the ferromagnetic magnons, and for power counting purposes it can be adequately represented by

$$
\chi^{\prime \prime}(\boldsymbol{k}, u)=\frac{1}{N_{\mathrm{F}} \lambda} \delta\left(u-\omega_{\mathrm{FM}}(\boldsymbol{k})\right)
$$

with

$$
\omega_{\mathrm{FM}}(\boldsymbol{k})=\text { const. } \times \lambda k^{2} / k_{\mathrm{F}}^{2}
$$

the ferromagnon frequency. Putting all of this together, and neglecting numerical prefactors, we obtain for the single-particle scattering rate due to ferromagnetic magnons

$$
\frac{1}{\tau(\boldsymbol{k})}=\frac{\lambda}{N_{\mathrm{F}}} \frac{1}{V} \sum_{\boldsymbol{p}} \frac{1}{\sinh \left(\omega_{\mathrm{FM}}(\boldsymbol{p}) / T\right)} \delta\left(\omega_{2}(\boldsymbol{k}+\boldsymbol{p})-\omega_{\mathrm{FM}}(\boldsymbol{p})\right),
$$

and for the electrical transport rate

$$
\begin{aligned}
\frac{1}{\tau_{\mathrm{el}}^{\mathrm{tr}}}=\frac{\lambda}{N_{\mathrm{F}}^{2}} \frac{1}{V^{2}} \sum_{\boldsymbol{p}, \boldsymbol{k}} \frac{p^{2} / k_{\mathrm{F}}^{2}}{\sinh \left(\omega_{\mathrm{FM}}(\boldsymbol{p}) / T\right)} \delta\left(\omega_{1}(\boldsymbol{k})\right) \\
\times \delta\left(\omega_{2}(\boldsymbol{k}+\boldsymbol{p})-\omega_{\mathrm{FM}}(\boldsymbol{p})\right) .
\end{aligned}
$$

Performing the integrals leads to the following results. For the single-particle relaxation rate on the Fermi surface, or the thermal resistivity, one finds

$$
\frac{1}{\tau} \propto \rho_{\text {th }} \propto \begin{cases}T e^{-\lambda^{3} / \epsilon_{\mathrm{F}}^{2} T} & \text { if } T \ll \lambda^{3} / \epsilon_{\mathrm{F}}^{2} \\ T \ln \left(T \epsilon_{\mathrm{F}}^{2} / \lambda^{3}\right) & \text { if } \lambda^{3} / \epsilon_{\mathrm{F}}^{2} \ll T \ll \lambda \\ T \ln \left(\epsilon_{\mathrm{F}}^{2} / \lambda^{2}\right) & \text { if } T \gg \lambda\end{cases}
$$

where we have omitted numerical prefactors as well as less-leading terms. For the electrical resistivity, the corresponding result is

$$
\rho_{\mathrm{el}} \propto \begin{cases}T\left(\lambda^{2} \epsilon_{\mathrm{F}}^{2}\right) e^{-\lambda^{3} / \epsilon_{\mathrm{F}}^{2} T} & \text { if } T \ll \lambda^{3} / \epsilon_{\mathrm{F}}^{2} \\ T^{2} / \lambda & \text { if } \lambda^{3} / \epsilon_{\mathrm{F}}^{2} \ll T \ll \lambda \\ T & \text { if } T \gg \lambda,\end{cases}
$$

We provide some brief comments regarding these results, a more complete discussion will be given elsewhere $\frac{52}{2}$ (1) The energy scales $\lambda^{3} / \epsilon_{\mathrm{F}}^{2}$ and $\lambda$ that lead to the three temperature regimes shown above emerge within the bare (Stoner-level) theory. It is not obvious how these scales become renormalized, and one therefore has to be careful when making quantitative comparisons with experiment. In particular, within the bare theory the regime $T \gg \lambda$ is not realizable since it implies $T>T_{\mathrm{c}}$; this may change within a fully renormalized theory. (2) The second result in Eq. (D4), $1 / \tau_{\mathrm{el}}^{\mathrm{tr}} \propto T^{2} / \lambda$, reproduces the result of Ueda and Moriya .54 We note, however, that this is not the true asymptotic low-temperature result; it is valid only in a pre-asymptotic temperature window. (3) The true asymptotic behavior for $T \rightarrow 0$ is exponential, rather than power-law, in $T$. This is due to the Stoner splitting of the conduction electrons; this effect was neglected in Ref. 54. In helimagnets the situation is qualitatively different, see Sec. IVB. 
1 I. E. Dzyaloshinsky, J. Phys. Chem. Solids 4, 241 (1958).

2 T. Moriya, Phys. Rev. 120, 91 (1960).

3 B. Lebech, J. Bernhard, and T. Freltoft, J. Phys. Cond. Matt. 1, 6105 (1989).

${ }^{4}$ Y. Ishikawa, K. Tajima, D. Bloch, and M. Roth, Solid State Commun. 19, 525 (1976).

5 L. Lundgren, O. Beckman, V. Attia, S. P. Bhattarcherjee, and M. Richardson, Phys. Scr. 1, 69 (1970).

${ }^{6}$ Y. Ishikawa, G. Shirane, J. A. Tarvin, and M. Kohgi, Phys. Rev. B 16, 4956 (1977).

7 O. Nakanishi, A. Yanase, A. Hasegawa, and M. Kataoka, Solid State Commun. 35, 995 (1980).

8 Y. Ishikawa and M. Arai, J. Phys. Soc. Japan 53, 2726 (1984).

9 B. Lebech, Recent Advances in Magnetism of Transition Metal Compounds (World Scientific, Singapore, 1993).

10 S. V. Grigoriev, S. V. Maleyev, A. I. Okorokov, Y. O. Chetverikov, and H. Eckerlebe, Phys. Rev. B 73, 224440 (2006).

11 S. Mühlbauer, B. Binz, F. Jonietz, C. Pfleiderer, A. Rosch, A. Neubauer, R. Georgii, and P. Böni, Science 323, 915 (2009).

12 C. Pappas, E. Lelièvre-Berna, P. Falus, P. M. Bentley, E. Moskvin, S. Grigoriev, P. Fouquet, and B. Farago, Phys. Rev. Lett. 102, 197202 (2009).

13 C. Pfleiderer, G. J. McMullan, S. R. Julian, and G. G. Lonzarich, Phys. Rev. B 55, 8330 (1997).

14 D. Belitz, T. R. Kirkpatrick, and T. Vojta, Phys. Rev. Lett. 82, 4707 (1999).

15 C. Pfleiderer, S. R. Julian, and G. G. Lonzarich, Nature 414, 427 (2001).

16 T. R. Kirkpatrick and D. Belitz, Phys. Rev. Lett. 104, 256404 (2010).

17 C. Pfleiderer, D. Reznik, L. Pintschovius, H. v. Löhneysen, M. Garst, and A. Rosch, Nature 427, 227 (2004).

18 Y. J. Uemura, T. Goko, I. M. Gat-Maleruanu, J. P. Carlo, P. L. Russo, A. T. Savici, A. Aczel, G. J. MacDougall, J. A. Rodriguez, G. M. Luke, et al., Nature Physics 3, 29 (2007).

19 S. Tewari, D. Belitz, and T. R. Kirkpatrick, Phys. Rev. Lett. 96, 047207 (2006).

20 B. Binz, A. Vishvanath, and V. Aji, Phys. Rev. Lett. 96, 207202 (2006).

21 U. Rößler, A. Bogdanov, and C. Pfleiderer, Nature (London) 442, 797 (2006).

${ }^{22}$ I. Fischer, N. Shah, and A. Rosch, Phys. Rev. B 77, 024415 (2008).

${ }^{23}$ M. Lee, W. Kang, Y. Onose, Y. Tokura, and N. P. Ong, Phys. Rev. Lett. 102, 186601 (2009).

24 D. Belitz, T. R. Kirkpatrick, and J. Rollbühler, Phys. Rev. Lett. 94, 247205 (2004).

25 D. Belitz, T. R. Kirkpatrick, and A. Rosch, Phys. Rev. B 73, 054431 (2006).

26 P. Chaikin and T. C. Lubensky, Principles of Condensed Matter Physics (Cambridge University, Cambridge, 1995).

27 D. Belitz, T. R. Kirkpatrick, and A. Rosch, Phys. Rev. B 74, 024409 (2006).

28 T. R. Kirkpatrick, D. Belitz, and R. Saha, Phys. Rev. B 78, 094407 (2008).

29 T. R. Kirkpatrick, D. Belitz, and R. Saha, Phys. Rev. B 78, 094408 (2008).
30 The term $(\boldsymbol{\nabla} \cdot \boldsymbol{M})^{2}$ or, equivalently (if combined with the $(\nabla \boldsymbol{M})^{2}$ term $),(\boldsymbol{\nabla} \times \boldsymbol{M})^{2}$, is not usually considered in the theory of classical Heisenberg ferromagnets. This term, as well as a stronger one, $|\boldsymbol{k} \cdot \boldsymbol{M}(\boldsymbol{k})|^{2} / \boldsymbol{k}^{2}$ in Fourier space, result from the classical dipole-dipole interaction, which in turn results from the coupling of the order-parameter field to the electromagnetic vector potential, see Ref. 55. These terms have small coefficients due to the relativistic nature of the dipole-dipole interaction, and therefore are usually neglected in the discussion of isotropic ferromagnets, despite the fact that they are renormalization-group relevant with respect to the critical Heisenberg fixed point. ${ }^{36,56}$ In the context of helimagnets, where many effects of qualitative interest are small due to the smallness of $g_{\text {so }}$, it is less obvious whether these terms can be ignored. However, for the field configurations we will consider they are not qualitatively different from the $(\nabla \boldsymbol{M})^{2}$ term, and we will neglect them. The question of a possible influence of the dipole-dipole interaction on the properties of the various phase transitions in a helimagnet is a different issue that we do not address in the current paper.

31 D. Belitz, T. R. Kirkpatrick, and T. Vojta, Rev. Mod. Phys. 77, 579 (2005).

32 M. L. Plumer and M. B. Walker, J. Phys. C: Solid State Phys. 14, 4689 (1981).

33 P. Bak and M. H. Jensen, J. Phys. C 13, L881 (1980).

34 M. B. Walker, Phys. Rev. B 40, 9315 (1989).

35 More precisely, the criterion is $b<b_{1} / 2-3 b_{1}^{2} q / c$. The reason for this correction is the $(\delta \pi)^{2}$ term in the free energy, Eq. (2.10a). We neglect this and other corrections that are of higher order in $g_{\text {so }}$. For instance, there are higher-order corrections to $H_{\mathrm{c} 1}$ as given by Eq. 2.13b).

36 S.-K. Ma, Modern Theory of Critical Phenomena (Benjamin, Reading, MA, 1976).

37 S. Sachdev, Quantum Phase Transitions (Cambridge University Press, Cambridge, 1999).

38 J. Hertz, Phys. Rev. B 14, 1165 (1976).

39 J. Zinn-Justin, Quantum Field Theory and Critical Phenomena (Oxford University Press, Oxford, 1996).

40 Strictly speaking, this is true only within a continuum model. In real magnets, the underlying lattice means that translational as well as rotational invariance is broken already in the disordered phase, and no truly massless modes will result from the order. However, since all of the phases of interest show some kind of helical order with a characteristic wave number $q$ that obeys $q a \ll 1$, with $a$ the lattice constant, the masses of the pseudo-Goldstone modes are exponentially small, see the discussion in Ref. 25. Any gaps in the excitation spectra are thus unobservably small, and we will ignore this effect.

41 D. Belitz, T. R. Kirkpatrick, A. J. Millis, and T. Vojta, Phys. Rev. B 58, 14155 (1998).

42 These arguments are the same as for cholesteric or smectic liquid crystals, see Refs. 26. 57. It should be noted, however, that the result is slightly different for electronic smectics and helical magnets, respectively, whereas for liquid crystals there is no difference between smectics and cholesterics in this respect, see Ref. 58 .

43 It is not entirely obvious that the dynamical part of the action density is $\left(\partial_{t} u\right)^{2}$, which leads to the square root in Eq. (1.1). Establishing this requires an explicit calculation, 
see Ref. 25.

44 A. H. Wilson, The Theory of Metals (Cambridge University Press, Cambridge, 1954).

45 J. M. Ziman, Principles of the Theory of Solids (Cambridge University Press, Cambridge, 1972).

46 G. Zala, B. N. Narozhny, and I. L. Aleiner, Phys. Rev. B 64, 214204 (2001).

47 It is remarkable that in this regime the difference between the single-particle relaxation rate and the electrical transport rate that is so characteristic of the Boltzmann theory of transport does not exist. From a scaling point of view one might speculate that this difference is not generic and will not be present in a strong-coupling theory that would be characterized by a single universal relaxation rate. Whether this is true, and if so, in what limit the Boltzmann result emerges, deserves an investigation.

48 J. Englert, H. Stark, L. Longa, and H.-R. Trebin, Phys, Rev. E 61, 2759 (2000).
49 L. Longa, M. Ciesla, and H.-R. Trebin, Phys. Rev. E 67, 061705 (2003).

50 M. Ciesla and L. Longa, Phys. Rev. E 70, 012701 (2004).

51 S. V. Maleyev, Phys. Rev. B 73, 174402 (2006).

52 D. Belitz and T.R. Kirkpatrick, unpublished results.

53 This can be seen, for instance, from Eq. (4.28) in Ref. 25, or from Eqs. (3.2) and (C1b in the present paper.

${ }^{54}$ K. Ueda and T. Moriya, J. Phys. Soc. Japan 39, 605 (1975).

55 D. Belitz and T. R. Kirkpatrick, Phys. Rev. B 81, 184419 (2010).

56 A. Aharony and M. E. Fisher, Phys. Rev. B 8, 3323 (1973).

57 P. G. DeGennes and J. Prost, The Physics of Liquid Crystals (Clarendon, Oxford, 1993).

58 T. R. Kirkpatrick and D. Belitz, Phys. Rev. B 80, 075121 (2009) 